\title{
Review of Near-Field Terahertz Measurement Methods and Their Applications
}

\section{How to Achieve Sub-Wavelength Resolution at THz Frequencies}

\author{
Aurele Joseph Louis Adam
}

Received: 22 February 2011 / Accepted: 28 June 2011 /

Published online: 28 July 2011

(C) The Author(s) 2011. This article is published with open access at Springerlink.com

\begin{abstract}
In the last decades, many research teams working at Terahertz frequencies focused their efforts on surpassing the diffraction limit. Numerous techniques have been investigated, combining methods existing at optic wavelength with $\mathrm{THz}$ system such as Time Domain Spectroscopy. The actual development led on one side to a resolution as high as $\lambda / 3000$ and one the other side to a video-rate recording. The purpose of this paper is to give an overview of the history of the field, to describe the different approaches, to give examples of existing applications and to draw the perspective for this research area.
\end{abstract}

Keywords Terahertz $\cdot$ Near-field $\cdot$ Subwavelength $\cdot$ Microscopy

\section{Introduction}

Over the last decade, optical near-field imaging has become an intense area of research. Many reasons for such popularity: the recent discovery of the extraordinary transmission of light through subwavelength structures [1], the invention of the near-field scanning optical microscope called NSOM [2, 3], or the irrepressible race to break the diffraction limit in order to design and fabricate smaller and smaller structures for the micro (nano)-electronics industry. Other branches of the electromagnetic spectrum are not to be outdone; Terahertz ( $\mathrm{THz}$ ) radiation in particular with its larger wavelength from $30 \mu \mathrm{m}$ to $2 \mathrm{~mm}$ corresponding to a frequency range spanning from $150 \mathrm{GHz}$ to

\footnotetext{
A. J. L. Adam ( $\otimes)$

TU Delft, Delft, The Netherlands

e-mail: a.j.l.adam@tudelft.nl
} 
$10 \mathrm{THz}$ and its properties contain many advantages for those who want to study near-field effects: structures can be done by classical optical lithography; metals like gold can be considered as perfect conductor; dielectric properties can be artificially engineered with the use of doped semi-conductors such as silicon. More important of all, a technique called Terahertz Time Domain Spectroscopy (THz-TDS) allows to measure the time-dependent electric field with an ultra-broad bandwidth, typically a decade or more $[4,5]$. Not only the intensity but also the phase and the polarization can be recorded with such system. Recently, large transients with peak electric fields of $108 \mathrm{MV} / \mathrm{cm}$ have even been reported opening the door to extreme non-linear studies [6]. These characteristics of the $\mathrm{THz}$ band explain why it draws much of the attention nowadays.

\subsection{Motivation and state of the art}

A previous review on $\mathrm{THz}$ microscopy was written by Yuan et al. [7], already seven years ago. Our motivation is to give an updated overview of this rich and dynamic subject which moved from microscopy to nanoscopy.

With the current techniques described below, subwavelength imaging at $\mathrm{THz}$ frequencies is already feasible. The highest resolution has been achieved by Huber et al. with a resolution of $\lambda / 3000$ at $2.54 \mathrm{THz}$ using a single frequency source and a scattering tip [8]. For broadband system based on single-cycle pulsed source, the resolution obtained is in the region of $\lambda / 600[9,10]$ with separate mapping of the different components of the electric near-field with its amplitude and phase. Commercial systems are currently available http://www.amo.de/?id=687. Generally the images are acquired via raster scanning and hence long to obtain. Fortunately video-rate imaging has recently emerged with a reasonable subwavelength spatial resolution of $\lambda / 4$ [11]. The $\mathrm{THz}$ near-field community is flourishing together with the possible applications in biology, nano-spectroscopy or plasmonics. As a result it sounds appropriate to offer to the reader, newcomer or expert, a complete overview of the field.

We will first give a panorama of the different techniques used in the past, based on apertures, waveguides, scattering properties of the a tip, direct detection and direct generation of the radiation. We will then demonstrate the advantages of these systems and their best results. In the following section, we will show the different possible applications using theses techniques with various examples. Finally, we will draw conclusions on the subject and give some suggestions for future research path for the field.

\subsection{Issues}

The permanent challenge when writing a review paper is to carry out an honest and careful investigation of other people's work, without repeating or paraphrasing what has already been done in the past and trying not to forget any relevant work. But it gives the rare opportunity to put on stage in one 
paper different methods, different approaches and to show how these not only compete but also profit to each other and to the subject in general. We hope to succeed in this quest.

We would also like to add that inherent to any imaging, especially in the near-field, there is always a debate on how we can reduce the fine features of the imaging techniques to a single parameter such as the "spatial resolution". Authors tend to use their own measurement technique to quantify their spatial resolution. Two techniques are commonly used, the "line spread function" (LSF) and the "edge response"; the line spread function is the response of the setup to a thin line-shape object across the light path. Similarly, the edge response is what one measures when a sharp straight discontinuity, an edge for example, is placed into the light path of the setup. Since a line is the derivative of an edge, the LSF is the derivative of the edge response in a linear system. Edge resolution is then defined as the lateral displacement with a change from 10 to $90 \%$ of the optical signal over an edge. Every author uses different methods (Rayleigh-type, edge, etc...) to give a value of their achievable resolution that may be disputable in regards to topological artifacts for example. To avoid any debate, we have decided to stick to the number given in the corresponding paper we are referring to and we will let the reader decide for himself on its accuracy.

\section{Techniques}

\section{$2.1 \mathrm{THz}$ sources}

In $\mathrm{THz}$ near-field imaging, as well as conventional $\mathrm{THz}$ imaging, the use of external THz source is mandatory. Many different sources will be introduced in this review, therefore to facilitate its reading, we would like to present briefly the different choices one has when building a $\mathrm{THz}$ near-field set-up.

The THz sources usually used in imaging are coherent and can be divided in two types: broadband sources and single frequency sources. Examples of the single frequency sources are direct multiplied source, backward wave oscillators (BWOs), far infrared laser such as $\mathrm{CO}_{2}$ based laser, p-Ge laser or recently the quantum cascade lasers based on GaAs/AlGaAs heterostructures. These sources can be run in a continuous mode $(\mathrm{CW})$ or/and pulsed mode. The main advantage of these source is their strong $\mathrm{THz}$ power output, reaching up to hundreds of $\mathrm{mW}$. The down side is that they may require cooling to operate and may need a sensitive detector such a Si bolometer to pick up the transmitted signal. The emitting frequency can be tuned, but the bandwidth depends strongly on the type of source. Therefore it limits the practicability for large bandwidth spectroscopy. Generally these sources are compact, reliable and have high stability; they will be ideal for imaging at a single frequency in a robust and small system.

On the other hand, the broadband sources are by definition broadband: they are typically based on ultrashort optical laser pulses which optically excite a 
medium producing the $\mathrm{THz}$ pulses via optical rectification or photoconductive antenna. These single-cycle pulsed source can cover a wide spectral range from 0.1 to $50 \mathrm{THz}$. With an adequate coherent detection scheme, based on electro-optical sampling or photoconductive antenna, the signal to noise ratio can reach 5000/1 despite the low average power levels, around tens of microwatts. They are ideal for time-domain terahertz spectroscopy (TDTS) to investigate the spectral response of materials. These systems are now commercially available but are rather expensive since it requires a femtosecond laser to operate. They are generally bulky and requires a large amount of optics, both for the femto-laser beam and the $\mathrm{THz}$ beam. These aspects are balanced by the broad spectral range they can generate enabling possible nearfield spectroscopy as we will show later.

The choices is therefore not easy to make: one as to decide the application he is looking for in order to determine which sources he will use. For further details on THz sources, we recommend the recent reference [12].

\subsection{Classical THz microscopy}

To study the response of a sample to a particular electromagnetic wave, one needs a source at that particular wavelength, an optical path in which the sample is located and a detector. The incident wave generated by the source is generally focused on the object. The wave interacts with the sample, is partly reflected, partly absorbed and partly transmitted. Usually the light (reflected or transmitted) is collected in the far field. For the THz regime, the procedure is similar. We present in Fig. 1 the typical set-up for far-field $\mathrm{THz}$ measurement. The source can be broad-band like in a TDS system or monochromatic as for a $\mathrm{THz}$ Quantum Cascade Laser (QCL) or a Gun oscillator. Because the $\mathrm{THz}$ wave behaves quasi-optically, the focusing is done using parabolic mirrors or Teflon lenses.

If the object is larger than the wavelength, we move the focus across it, record for each position the transmitted/reflected radiation and stitch side by side the results. We obtain a pixelated image of the sample at the desired wavelength. If the object is smaller than the wavelength, the incident wave will seem to be unperturbed by the presence of the object when recorded in the

Fig. 1 Typical THz far field set up: a THz source is emitting a wave collected by a parabolic mirror, then focused on the sample via an other parabolic mirror. The transmitted signal is collected via two more mirrors and finally focused on the $\mathrm{THz}$ detector.

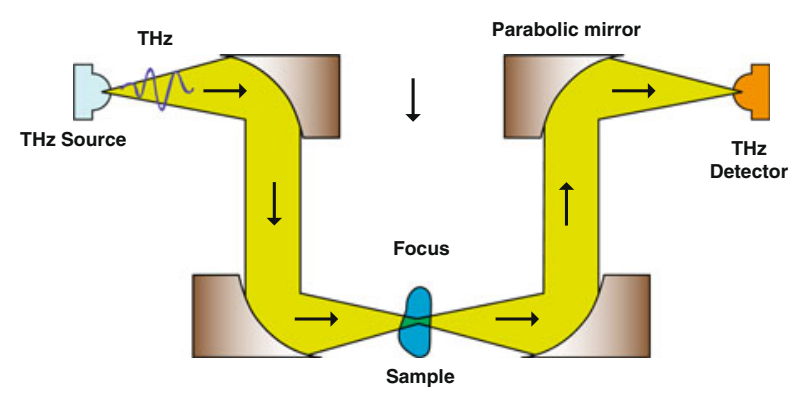


far field. This can be shown using a simple Mie scattering approach: a sphere made of a perfectly conducting material is placed on the path of an incident plane wave. We have plotted in Fig. 2 the time shot of the same incident plane wave for two different sizes of the sphere: on the left, the wavelength is larger than the diameter of the sphere; on the right, the wavelength is comparable to the sphere diameter. One can see that far away from the large sphere there is a clear perturbation of the plane wave; for the small sphere, after less then a few wavelengths, there is no noticeable change anymore. Nevertheless, if one places a detector able to measure the field at a distance smaller than the wavelength, also called the near-field, the observer will be able to "notice" the sphere. Therefore if ones wants to observe a small object or small changes in an object, it is needed to perform the measurement in the near-field. Thus all the interest of near-field imaging. The challenge is how to measure the field in such close proximity of the object without perturbing the field, with a good signal to noise ratio and with a high resolution.

An other way to approach the problem comes from conventional imaging: when focusing an incident wave with a lens to a small spot, the diffraction limit or Rayleigh diffraction makes that the formed spot is not a point as expected but an Airy disk [13]. This disk has a radius $d$ to first zero equal to $1.22 \lambda N$ where $N$ is the ratio of the focal length $f$ to the diameter $D$ of the entrance pupil. $N$ is known as the numerical aperture of your optical system and $\lambda$ is the wavelength of the incident wave. When imaging two different points sources, they will give two different Airy disks in the image plane which may overlap if the two sources are too close: in other words, the two sources will not be distinguishable in the image.

The problem encountered at optical frequencies is the same at $\mathrm{THz}$ frequencies. To give an order of magnitude, for a frequency of $1 \mathrm{THz}$, corresponding to a wavelength of $300 \mu \mathrm{m}$, the minimum resolution will be approximately half of the wavelength thus $150 \mu \mathrm{m}$.

In this section, we would like to give an overview of the different techniques that have been developed during the last two decades to reach subwavelength resolution for the $\mathrm{THz}$ near-field measurement. We've tried to group the different techniques in five categories based on their main operation principle:

Fig. 2 Example of Mie scattering: main electric field component at a given time around a metallic sphere of $100 \mu \mathrm{m}$ (left) and $600 \mu \mathrm{m}$ diameter (right). The incident plane wave travels from top to bottom at a frequency of $1 \mathrm{THz}$. Dark represents negative field and white positive field.
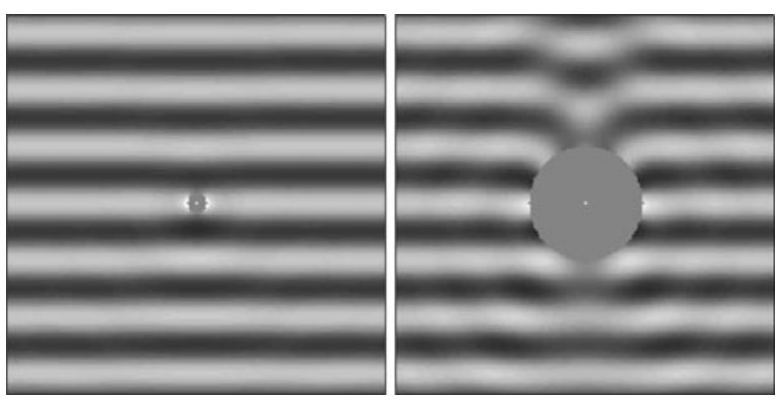
the confocal approach, the apertures principle, the scattering method, the guided mode on wire method, and the direct detection method.

\subsection{Confocal THz microscopy}

The concept of confocal microscopy at optical wavelengths is pretty recent, despite its simplicity, and it is widely used nowadays to improve image resolution in optical microscopy. For more on the history of the discovery, see [14]. The idea is to position a small aperture at the focal plane of a microscope objective. Because the aperture is placed at the focal plane, it will block rays coming from regions of the object that do not coincide with the conjugate image. Not only the lateral resolution is improved but also the depth resolution. The concept has been recently applied at $\mathrm{THz}$ frequency, using a traditional TDS system, with two extra parabolic mirrors, creating a second focus in the $\mathrm{THz}$ path, as seen in Fig. 3 [15]. The authors place an iris of diameter $1.2 \mathrm{~mm}$ at the second focus. They demonstrate an improvement of the lateral resolution $(\lambda / 4)$ without any loss in frequency bandwidth.

This approach brings slightly better resolution at the cost of alignment hassles but it has the advantage of its simplicity.

\subsection{Aperture}

The simplest and therefore straightforward way to reduce the spatial resolution is to reduce the size of the detector system and to bring the detector close enough to the object under examination. To do so, the use of an aperture is one of the options. The field of interest will be squeezes in the aperture and only what could come in and out of this aperture will be detected: the spatial resolution is reduced to the size of the aperture. Main inconveniences of the method is that it filters the signal due to the cut-off of the waveguide created by the aperture and considerably reduces the signal. Generally the apertures are made as a simple hole in a metal. More complex structures around or before the aperture can enhance the transmitted signal.

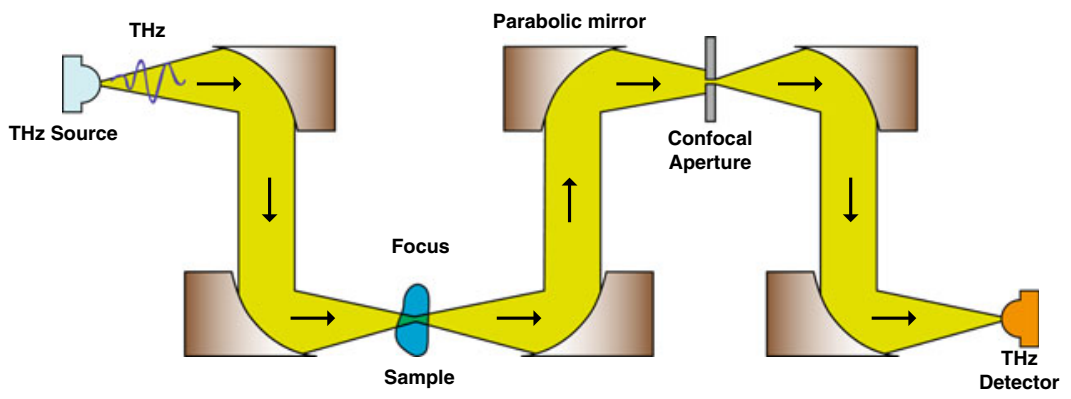

Fig. 3 Confocal microscopy, applied to a THz set-up shown in Fig. 1. Two parabolic mirrors and an aperture placed at the second focal plane have been added [15]. 
Basic apertures The first example of a real aperture used for a scanning microscope concerns in the microwave region at $10 \mathrm{GHz}$, by Ash et al. in 1972 [16]. Following this example, Mitrofanov et al. demonstrated in 2000 the same principle at $\mathrm{THz}$ frequencies [17]. For this experiment, the authors define a square aperture of $50 \times 50 \mathrm{\mu m}^{2}$, made from a $600 \mathrm{~nm}$ gold layer deposited on a Gallium Arsenide (GaAs) wafer as show in Fig. 4a. The detector, a photoconducting antenna, is placed just $4 \mu \mathrm{m}$ below the aperture, right in its center. It measures the electric field of the transmitted $\mathrm{THz}$ pulses in the near-field zone of the aperture. To collect more $\mathrm{THz}$ light, a cone or a silicon lens can be placed on top of the aperture [18]. As explained above, the aperture will filter the low frequencies depending on the size of the aperture. This cut-off frequency can be easily calculated using waveguide theory and has been demonstrated experimentally at $\mathrm{THz}$ frequencies [19]. For example for a circular hole for example, the cut-off frequency of the dominant $\mathrm{TE}_{11}$ mode should be equal to $1.841 c / \pi d$ with $d$ the diameter of the aperture and $c$ the speed of light in vacuum. So for a hole of $100 \mu \mathrm{m}$ diameter, the cutoff frequency is $1.76 \mathrm{THz}$ or $170 \mu \mathrm{m}$. One can see here the irony of using an aperture for subwavelength resolution. Of course this cutoff is only existing for waveguides longer than several wavelength. If the waveguide is short, many non propagating modes will be able to reach the end of the waveguide and will be diffracted by the hole. This is why Mitrofanov could use such a small aperture and still measure a signal.

So the first question regarding the use of an aperture is how far one can go in reducing the aperture size? The theoretical study of light going through a circular hole is well described in two papers, one by Bethe [20], one by Bouwkamp [21]. Below the cut-off frequency, the amplitude of the electric field after the aperture for a infinitely thin metallic screen linearly decreases with $d$, where $d$ is the diameter of the aperture, see Fig. 5a with a plot of the main component of the electric field, for different distances below the aperture, of which the diameter varies from 3 to $1,000 \mu \mathrm{m}$ for a fixed

Fig. 4 a Mitrofanov's approach of using an aperture with the detector just below it [17]; b Aperture with bull's eye structure: the $\mathrm{THz}$ beam is focused on the corrugated front surface creating surfaces waves which will leak into the aperture, enhancing the $\mathrm{THz}$ transmitted at the designed frequency [22].

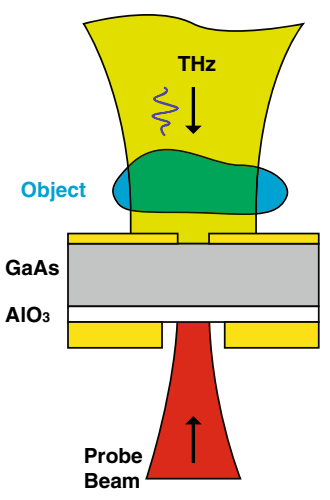

(a)

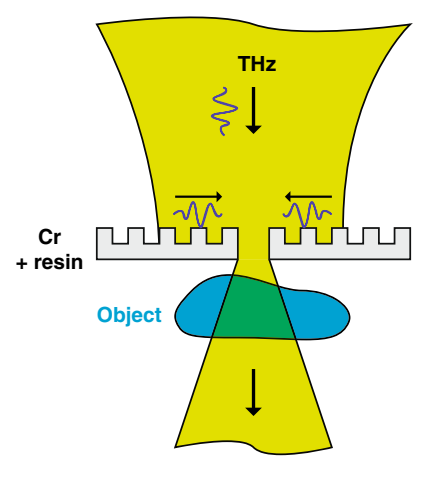

(b) 
wavelength of $300 \mu \mathrm{m}(1 \mathrm{THz})$. One can see that this linear dependance is only valid inside the hole or for distance smaller than the hole diameter: $z<d$. For larger distances, the Bouwkamp model clearly follows a cubic power dependence on the aperture diameter, as observed by Mitrofanov [17]. So decreasing the aperture size while keeping the same distance to the hole will inevitably decrease the signal to noise ratio and make the signal more difficult to measure. Be aware that for an aperture size larger than the wavelength, the calculation of Bouwkamp is not valid anymore and such a case has not been represented in Fig. 5a.

The second question is how far the detector should be from the hole to still be able to detect an useful signal. One expects the hole to diffract the light so much that no appreciable signal can be detected in the far field. Mair et al. have shown experimentally that the signal transmitted from an aperture of diameter $d$ decays exponentially with the distance to the aperture $z$ :

$$
E_{x} \propto e^{-z / t d}
$$

with $t \approx 0.65$ a numerical factor, independent of the wavelength as long as $\lambda \gg z$. This is only valid for the field close to the aperture as seen in Fig. 5b: we present the calculation from Bouwkamp of the main component of the electric field after a subwavelength hole of different diameters $(5,10,50$ and $100 \mu \mathrm{m})$ for a wavelength of $300 \mu \mathrm{m}(1 \mathrm{THz})$. As one can see, the exponential dependence is only valid for distance smaller than half of the wavelength and for small diameter. For larger distances, the decay is much slower according to the Bouwkamp model: it goes as $d^{3} / z^{2}$ for $z \gg d$. For large diameter, the electric field decreases very slowly with the distance.

As a conclusion of these calculations, we should define the near-field as the field in a region within not a wavelength of the object but in a region within the characteristic dimension of the object. Typically, if you want to measure the near-field from a $5 \mu \mathrm{m}$ holes, your detector should be placed at least $1 \mu \mathrm{m}$

(a)

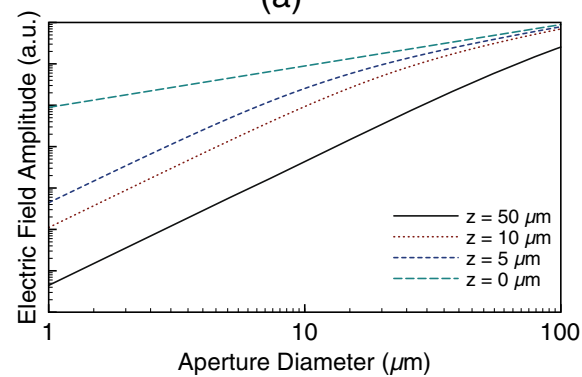

(b)

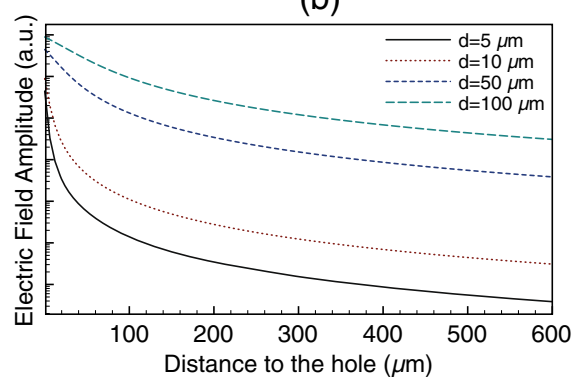

Fig. 5 Dependence of the main component of the near-electric field diffracted by a round hole for a $300 \mu \mathrm{m}$ wavelength incident light, using the Bouwkamp model: a aperture diameter $d$ dependence for different distances below the center of the hole $[18,21]$; $\mathbf{b}$ dependance of hole distance for different hole diameters [21, 23]. 
from the hole to detect the proper near-field whatever your wavelength is in the case it is larger than the hole diameter.

The Bouwkamp model describes a hole bored in a infinitely thin opaque screen. In reality, an infinitely thin screen is never opaque to the incident radiation. Any metal presents a finite penetration depth: gold, for example, has a $50 \mathrm{~nm}$ penetration depth in the $\mathrm{THz}$ range. Which means that some $\mathrm{THz}$ radiation is transmitted through the sheet layer, and its amplitude can be comparable to that of the $\mathrm{THz}$ radiation diffracted by a tiny hole. Therefore to get more light from the hole than from the direct transmission through the screen, the thickness of the screen has to be optimized [18]. Using the optimized aperture described above, Mitrofanov could demonstrate a spatial resolution of $7 \mu \mathrm{m}$ for a $5 \mu \mathrm{m}$ aperture at frequencies from 0 to $2 \mathrm{THz}(\lambda / 70)$ using a standard TDS set-up.

We have understood that the resolution obtained with this technique is strongly related to the size of the aperture but reducing the size of the aperture, also reduces the signal which goes through. To overcome this situation, a computational trick can be used: the Differential Near-Field Scanning Optical Microscopy (differential NSOM) [24]. In this case, an aperture with a size comparable to that of the incident wavelength is scanned in the nearfield of the object of interest. The transmitted signal is recorded in the far field. The recovery of the near-field image is accomplished by performing a numerical second order derivative $\frac{\partial^{2}}{\partial x \partial y}$-operation of the image acquired by the detector. This technique has been successfully used by Degl'Innocenti et al. with a Terahertz Quantum Cascade Laser (THz-QCL) as a source emitting at $105 \mu \mathrm{m}$ [25]. They have reached a $\lambda / 10$ resolution with a $100 \mu \mathrm{m}$ square aperture.

Corrugated apertures The main issue with a tiny hole is the coupling between the incident $\mathrm{THz}$ light and the hole area. Typically, the incident wave has a gaussian beam shape at the focus, with a much larger width than the hole diameter. A large mismatch has to be expected. In order to enhance the signal coupled into the aperture, many different schemes have been introduced. The use of a bull's eye structure, i.e. a single subwavelength aperture surrounded by concentric periodic grooves in a metal plate, is one of them [26]. The principle is given in Fig. 4b: the incident $\mathrm{THz}$ wave is resonantly coupling to the surface wave modes caused by the periodic grooves. These surface waves concentrate at the center of the structure, where the hole is placed. More THz light is then coupled into the hole and, therefore, can be diffracted to the other side. Ishihara et al. have made a $100 \mu \mathrm{m}$ diameter aperture on a rather thick plate $(60 \mu \mathrm{m})$. A thick plate is not advocated because it reduces the amount of light reaching the other end of the aperture. But this is necessary to pattern the 10 concentric periodic grooves of $100 \mu \mathrm{m}$ width and $20 \mu \mathrm{m}$ depth with $200 \mu \mathrm{m}$ periodicity. They've obtained a 20-fold enhancement of the transmitted $\mathrm{THz}$ light compared to the bare aperture at the designed frequency of $1.5 \mathrm{THz}$ dictated by the periodicity of the grooves. One can see the drawback of this 
technique: it considerably reduces the bandwidth of the system. Nevertheless, they reach a $50 \mu \mathrm{m}$ resolution $(\lambda / 4)$ for the planned frequency.

Instead of improving the coupling of the incident $\mathrm{THz}$ wave to the aperture one can concentrate efforts into extracting more light from the aperture exit. For example, bringing an apex for example to the aperture will provide better re-radiation of the leaky waves and then higher transmission than with a simple round aperture. Ishihara et al., for example, present a combination of a bull's eye structure with a bow tie antenna aperture leading to an improved spatial resolution of $\lambda / 17$ [22].

We have seen that the signal diffracted by the aperture is stronger when the detector is close to output of the aperture. In 2008, Kawano et al. used a $8 \mu \mathrm{m}$ aperture in combination with, few nm below, a two-dimensional electron gas in a GaAs/AlGaAs heterostructure chip as $\mathrm{THz}$ detector [27]. The detector has to be cooled at cryogenic temperature $(13 \mathrm{~K})$ for good operation. They show that by placing the detector much further away $(10 \mathrm{~cm})$ no $\mathrm{THz}$ signal was detected through the aperture, proving that only evanescent waves leaked out from such a small aperture. In addition, they have placed a near-field probe, on the back of their aperture, in order to couple out more efficiently the evanescent field escaping the hole. In their measurements, they've shown that only when the probe is added to the aperture, they can measure a signal with their detector at the aperture. Finally, they've reached a spatial resolution of $8 \mu \mathrm{m}$ for $\lambda=214.6 \mu \mathrm{m}(\lambda / 24)$ using a THz gas laser.

Waveguides Beside the corrugation to improve the coupling of the incident wave to the aperture, other methods based on guiding the wave into the aperture have been investigated in the past. The first example is given by Hunsche et al.: they use a tapered aperture (TA) made in a hollow tube, analogous to the tapered fibers used in near-field scanning optical microscopes [28]. They demonstrate a spatial resolution of $55 \mu \mathrm{m}(\lambda / 4)$ for a bandwidth up to $1.25 \mathrm{THz}$ with their $50 \times 80 \mu \mathrm{m}$ elliptical aperture. Due to the tapered geometry, they could not observe a strong cut-off coming from the aperture for the low frequency. Interestingly, because of the elliptical shape of their aperture, they note a reduced resolution when scanning the object along the major axis of the aperture. Similar results have been achieved by Nguyen et al. using a conical aperture in $25 \mu \mathrm{m}$ thick stainless steel foils [29]. They compared the signal transmitted from their TA with an opening diameter of $1.9 \mathrm{~mm}$ and an exit aperture diameter of $420 \mu \mathrm{m}$ to the signal transmitted by two cylindrical apertures of $1.9 \mathrm{~mm}$ and $420 \mu \mathrm{m}$. They show a 7-fold increase in the $\mathrm{THz}$ electric field concentration. The amplitude concentration factor varies inversely with the diameter of the exit aperture. Unfortunately, reducing this parameter will also increase the cut-off frequency and therefore reduce the overall transmitted signal. They further improve their TA by adding annular groves on its entry side. For the design frequency of $0.3 \mathrm{THz}$, they reach $35 \%$ enhancement relative to the same TA without the groves. 
In order to compensate the loss due to the aperture, Staats et al. studied a conical parabolic waveguide with an antenna feed coupled with the beam of a synchrotron, delivering more input power than in the conventional way to compensate the loss from the aperture [32]. First they simulate such an aperture, then they investigate a scaled model at $\mathrm{GHz}$ frequencies. Finally they realized a prototype using micromechanics for the range between 0.1 and $1 \mathrm{THz}$. The results were a large field intensity at the tip of their cone, able to destroy the tip in some cases. This type of antenna-coupled aperture shows great potential.

To avoid the cut-off due to the circular aperture, Awad and Cheville used instead parallel-plate waveguides (PPWG) known for not presenting any cutoff for a particular mode [30]. They add a cylindrical lens to collimate the $\mathrm{THz}$ beam to the $\mathrm{TM}_{0}$ mode of the waveguide and placed the system directly after their object as shown in Fig. 6. The slit between the two plates is in the order of $100 \mu \mathrm{m}$ and the length of the waveguide is $25 \mathrm{~mm}$. They obtain a $\lambda / 4$ resolution at $0.6 \mathrm{THz}$, an improvement with respect to what is obtained with only the two cylindrical lenses.

Mittleman's group took also advantage of the parallel-plate waveguides to avoid cut-off and improved the idea by tapering the PPWG [31]. The output facet of their PPWG is adiabatically tapered in width and in separation between the plates to values of $10 \mu \mathrm{m}$ and $18 \mu \mathrm{m}$, respectively. They show that the taper does not distort the time-domain signal measured at the output of the PPWG. The field profile at the output strongly depends on the plate separation: the confinement factor defined as the ratio of the total $\mathrm{THz}$ energy within the waveguide to the total $\mathrm{THz}$ energy in the plane of the output facet increases exponentially towards 1 when the plate separation goes down to zero. They succeed to confine the $\mathrm{THz}$ beam to an area with a lateral resolution

Fig. 6 Above Untapered parallel-plate waveguide [30]; Below tapered parallel-plate waveguide [31].
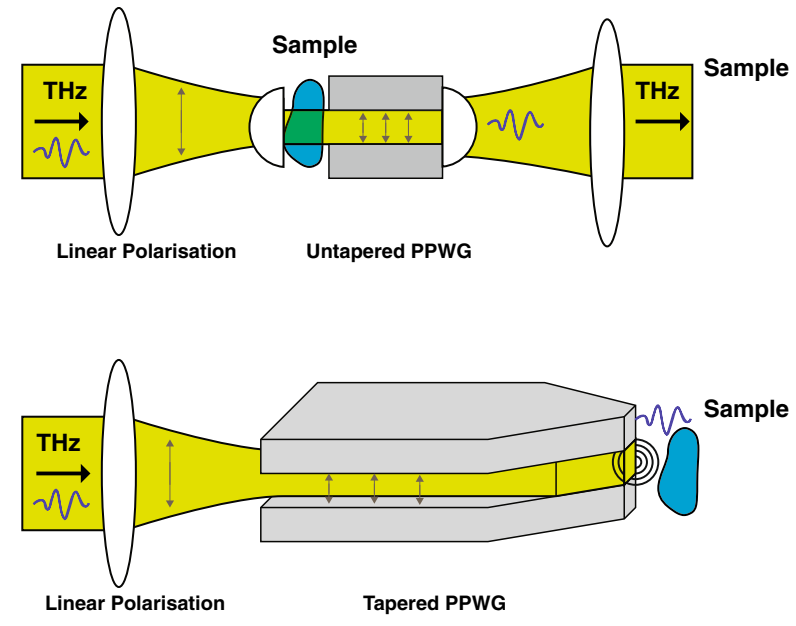
related to the plate width at the output $(\lambda / 260)$ at their peak amplitude $(0.12 \mathrm{THz})$.

The group of Kadlec in Prague has developed a waveguide based on a parallel plate with a low loss dielectric in between [33]. They sharpen a pyramidal tip on a silicon needle bar by mechanical polishing. The end facet has an area of $30 \times 40 \mu \mathrm{m}^{2}$. Two opposite side facets of the pyramid are coated to create the metallic waveguide. The incident $\mathrm{THz}$ beam is focused on the entrance facet as shown in Fig. 6b. It propagates inside the waveguide and gets gradually confined between the two metallic sides. Because of the constant wave impedance along the tip, the pulse is reflected only than at the end facet. This reflected pulse then propagates back, exits the waveguide and is finally detected. It contains information on the dielectric properties of whatever is close to the end facet. Using a complex algorithm, the authors [34] can retrieve this information. For the measurement itself, they obtain about $20 \mu \mathrm{m}$ resolution corresponding to $\lambda / 200$ at $80 \mathrm{GHz}$.

Other types of dielectric probes have also been investigated in the millimeter-wave regime [35]. Tapering a Teflon rod to a cone with a final diameter of $1.7 \mathrm{~mm}$ makes a rather simple probe where the $\mathrm{THz}$ can be guided as depicted in Fig. 7c. A cross-slit of $3 \mathrm{~mm}$ width could be imaged, equivalent in size with their used wavelength $(93.5 \mathrm{GHz})$.

Finally, Chiu et al. have presented an other use of the bull's eye structure in combination with a subwavelength polyethylene (PE) fiber used as a $\mathrm{THz}$ guiding fiber optimized for $312 \mathrm{GHz}$ [36]. This is similar to what is done in near-field optical microscopy, when a fiber will bring the light directly in close contact with the sample. In Chiu's case, the $\mathrm{THz}$ wave is focused into the fiber, which has $10 \%$ loss for a bend of $2^{\circ}$. A corrugated structure is placed at the end of the PE fiber to enhance the coupling to the

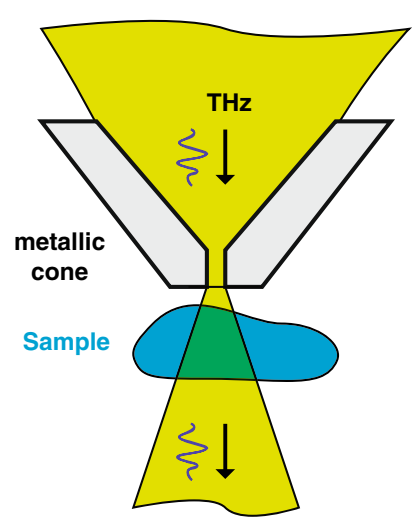

(a)

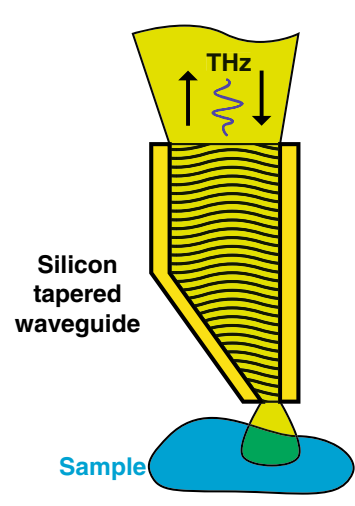

(b)

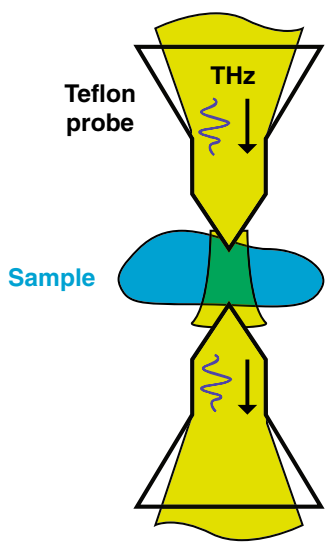

(c)

Fig. 7 a Tapered aperture made with a conic shape [28]; b dielectric waveguide with metallic walls on opposite side [33]; c dielectric probe-tip [35]. 
aperture. This way, the system with fiber/corrugated hole makes a perfect probe for near-field measurement. The authors achieve a spatial resolution of $210 \mu \mathrm{m}(\lambda / 4)$ and applied the technique to breast tissue analysis (see Section 3.6).

Other configurations for waveguides have been discussed, like metal/ dielectric waveguides with optimum shapes [37] but they have not yet been fabricated and measured.

\subsection{Scattering method}

It is well known that metallic edges or sharp extremities are scattering off any light incident on it [38-40]. The idea is to place a sharp tip, smaller than the wavelength directly at the vicinity of the sample. An incident beam is then focused onto the sample. The tip will pick up the near-field present at its end and scatter it off in the far field. The scattered field is then collected in the near-field or in the far field as we will see further. This method provides a subwavelength resolution in the immediate neighborhood of the tip apex. To separate the near-field information scattered by the tip end from the rest, the metal tip is generally vibrated at a high frequency $\Omega$, followed by lock-in detection at this frequency or at higher harmonic frequencies $n \Omega$ with $n$ equal to two or three. In this section we will introduce the various methods using a scattering tip. The interaction tip/sample will be discussed in Section 3.4.

Dynamic scatterer Real apertures have the default to be set once for all. If one wishes to have more control on the aperture itself while doing the measurement, an aperture which could open at any time or that could change its area would be an asset. In order to achieve this, the general idea is to create a dynamic aperture. Two kinds of aperture can be imagined: the first one, most obvious of all, is the positive aperture: a aperture is created in a opaque medium in the path of the light beam. This method hasn't been developed in the $\mathrm{THz}$ regime as far as we know.

The second way is the inverse of the previous one and it is based on a negative aperture. This time, an opaque area smaller than the wavelength is introduced in the path of the $\mathrm{THz}$ beam; it acts as a scatterer for the $\mathrm{THz}$ radiation. If the scatterer presence is modulated externally, using a lock-in configuration, we can read information only from this area. Developed in the mid-infrared (IR) [42], this method has been transposed in the $\mathrm{THz}$ regime. Usually it is classified as an dynamic aperture, but we have decided to put it in the scattering section due to its nature which is closer to a scatterer than an aperture.

To realise the negative aperture, a semiconductor material such as GaAs is commonly used [41]. A sketch of the setup is given in Fig. 8. The scatterer is produced via photon excitation of free carriers in the semiconductor, generated by the use of a focused optical gating beam originating from the main femto-second laser which is also used to generate and record the $\mathrm{THz}$ pulse. The photo-carriers have a recombination time much longer than the 
Fig. 8 Dynamic scatterer: an optical gating beam is focused on the GaAs plate that locally blocks the THz beam. The measured $\mathrm{THz}$ signal is proportional to the fluctuation of $\mathrm{THz}$ transmission [41].

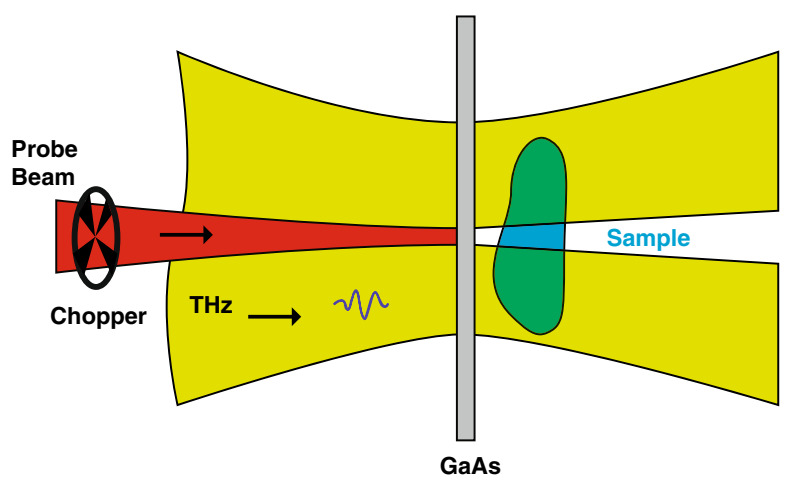

THz pulse duration but shorter than the time interval between two consecutive pulses from the femto-laser. This means that if the pump pulse is reaching the semiconductor before the $\mathrm{THz}$ pulse arrives, it will become opaque in the area of the focus for the complete duration of the pulse. The modulation is obtained by chopping mechanically the optical gating beam. Short carrier lifetimes and a small diffusion length are key to this type of near-field measurement. These can be addressed via the choice of semiconductor materials and its appropriate doping. Chen et al. obtained a $36 \mu \mathrm{m}$ resolution at $0.9 \mathrm{THz}(\lambda / 10)$ for a gating beam focal size estimated to $22 \mu \mathrm{m}$ on GaAs [43]. Gompf et al. [44] are using a continuous wave source and a Golay cell as detector. They obtain similar results using silicon as semiconductor and a green laser diode as optical gating beam with $100 \mu \mathrm{m}$ resolution at $0.45 \mathrm{THz}(\lambda / 6)$.

The advantages of such an approach are the following: it doesn't require a complicated set-up, the detector nor the source have to be in the vicinity of the sample, only the dynamic aperture should be nearby. As for the real aperture, the resolution is increasing with the decrease of the spot size of the optical gating beam but this considerably lessens the modulation of the measured $\mathrm{THz}$ beam and thus it signal to noise ratio. The local temperature increases at the focused spot on the semiconductor can also obscure the modulation due to the local photocarriers and, therefore, reduce the signal to noise ratio. Increase the beam diameter reduce these effects but also reduces the resolution. A comprise has to be found between a high resolution and a good signal.

In order to avoid this inconvenience when using the negative aperture, other methods have been investigated and most of them were inspired by the work done at optical and near-infrared wavelength on Apertureless Near-field Scanning Optical Microscopy abbreviated to ANSOM.

Metal tip-near-field collection The ideal scatterer to study and therefore to use would be a infinitely small sphere as in Section 2.2. The closest geometry to sphere, that one can easily manipulate, is a sharp tip. Its extremity, or apex would be the equivalent of the sphere. It makes use of the evanescent fields that exist only near the sample and will scatter them. The shaft of course can be 
bothersome but is required to actually "control" the position of the apex over the sample. This geometry is widely used at optical wavelengths to achieve subwavelength resolution. It works the same way at $\mathrm{THz}$ frequencies. To be a good scatterer, the tip is usually made from metal, because it can be consider as a perfect conductor. Once such tip is placed above the sample, the $\mathrm{THz}$ scattered by the tip apex has to be collected in some way. Because the scattered field is weak as compared to the field incident on the sample, the best way to reach an acceptable signal to noise ratio is to collect it in the near-field, i.e. close to the apex itself.

Such strategy has been first employed by Planken's group in 2002 [45]. As a detector they used an electro-optical (EO) crystal such as GaP on which they deposit the sample. The tip is placed above the sample illuminated by an incident THz beam as shown in Fig. 9a. A synchronized probe pulse, derived from the same laser that generates the THz pulse, is focused in a $20 \mu \mathrm{m}$ spot size at the interface sample/crystal, initially counter propagating with respect to the $\mathrm{THz}$ pulse. A coating designed to reflect the probe beam and to be transparent to the $\mathrm{THz}$ is deposited at the interface. The EO crystal is chosen judiciously so only one of the components of the electric field can be detected: the same as the incident one or one of the two perpendicular components. Indeed, the tip is scattering in every direction and therefore creates components of all polarizations. Part of this field will be non propagating. The appropriate choice of crystal orientation will make the detection system blind to the incident field and only the non-propagating component created by the presence of the tip end will be detected. In this method, the resolution is determined by the spot diameter of the probe laser, not by the incident $\mathrm{THz}$ beam size which will bring the resolution down to the $\mu \mathrm{m}$ range for the metallic tip they use.

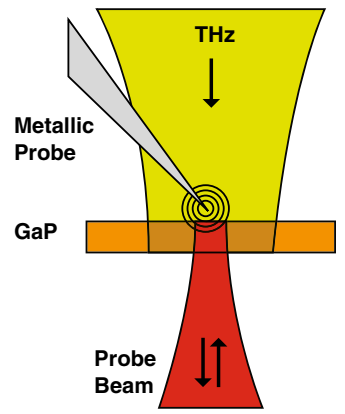

(a)

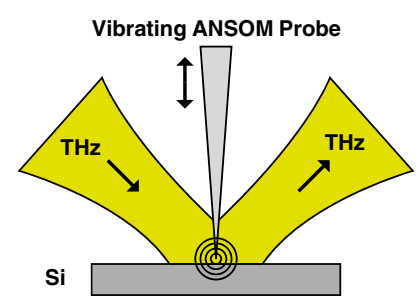

(b)

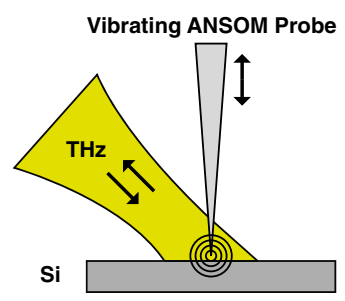

(c)

Fig. 9 Schematic for the tip scattering method: a Collection in the near-field: an EO crystal is placed underneath the tip. Only the component of the electric field parallel to the probe propagation direction will be measured [45]. b Collection in the far field: the forward scattered field is directly measured in the far field. Direct reflection is filtered thanks to the modulation of the tip/sample distance [46]. c The back scattered field is collected in this configuration [8]. 
Van der Valk et al. presents the measurement of the near-field component of the scattered electric-field at the end of a metallic tip. The smallest spot size achieved has a diameter of $8 \mu \mathrm{m}$ for a bandwidth from 0.1 to $1.5 \mathrm{THz}(\lambda / 100)$. This result shows a very good signal to noise ratio due to the proximity of the detector and the scattering structure.

Metal tip-far-field collection Contrarily to the previous detection scheme, the collection of the scattered field can also be done in the far field. Many of these scattering measurements have been successfully achieved in the optic and the infrared domain [40,47]. While most of these measurements are done in a back-scattering configuration, the first report on far-field collection at $\mathrm{THz}$ frequencies was done by Chen et al. with the detection module placed in forward direction as shown in Fig. 9b [46]. They used a THz-TDS set-up with EO sampling detection and also bolometric detection. The scattered field is $0.4 \%$ of the incident one. As tip, they first used a tungsten needle with $1 \mu \mathrm{m}$ tip radius, fixed at $200 \mathrm{~nm}$ above the sample, with no oscillation. They report a $3 \mu \mathrm{m}$ resolution at $2 \mathrm{THz}(\lambda / 50)$. When using a smaller tip, like the one found in Atomic Force Microscope (AFM), with a $100 \mathrm{~nm}$ radius, submicron resolution is achievable. They claim a $150 \mathrm{~nm}(\lambda / 1000)$ resolution measured on a semi-insulating silicon with metallic stripes of nominally $10 \mu \mathrm{m}$ width. Using shear-force control on their tip and oscillatory motion of the tip in the order of $1 \mathrm{~nm}$, they decreased the tip/sample distance to $20 \mathrm{~nm}$ [48]. Their results will be discussed in Section 3.4.

A similar set-up based on a fiber-coupled broadband $\mathrm{THz}$ system used for generation and detection in a ANSOM setup has been built in Mittleman's group [49]. They vibrate the tip perpendicularly to the sample surface with an amplitude of about $750 \mathrm{~nm}$ and lock their measured signal to this frequency. They succeed to measure with a micron-scale spatial resolution metallic domains in inhomogeneous VO2 thin films.

Following their work done in the near-infrared regime [50], Keilmann's group at the Max Planck Institute of München developed its own approach: they collect the scattered field in the back-scattered direction placing a cantilevered $\mathrm{W}$ tip, used in tapping AFM mode, at the focus of a paraboloid mirror as shown in Fig. 9c [51]. They collect the scattered field at about $90^{\circ}$ sideways. In order to suppress the background scattering coming from the shaft and not the tip apex, they develop a method based on demodulating the detector signal at a low harmonic of the tapping frequency. They show that the first and second-order demodulated scattering spectra can be recorded. The first order one is still contaminated by the background scattering, while the second order one is not. They do not report a value for the resolution but show the amplitude contrast of their measured signal when the tip is over two samples of silicon, doped differently. In this set-up, they generate a large bandwidth THz pulse via a GaAs emitter with Ti:sapphire laser pulses, and detected it by a standard EO setup. In 2008, the same group changes the configuration of their set-up [8]. They use a $\mathrm{CW} \mathrm{THz} \mathrm{laser} \mathrm{in} \mathrm{combination}$ with a bolometer, and introduce an interferometric arm via a beam splitter. 
Fig. 10 Emission scatterer example: the $\mathrm{THz}$ is directly generated by focusing the pump beam on the InAs crystal beneath the ANSOM probe. An AC voltage is applied to lock the detected signal [53].

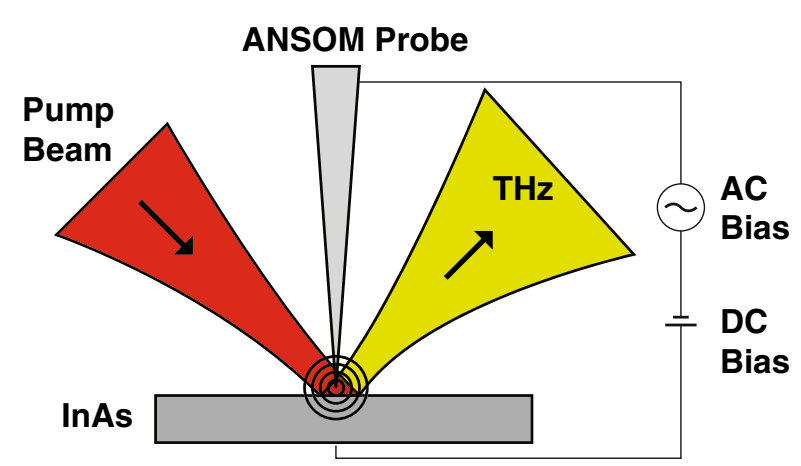

They continue to demodulate their signal at harmonics frequencies. They were able to record simultaneously the topography via the AFM tip and perform mid-IR measurement with the same configuration with a $\mathrm{CO}_{2}$ laser illumination at $28 \mathrm{THz}$. They show $40 \mathrm{~nm}(\lambda / 3000)$ spatial resolution at $2.54 \mathrm{THz}$. The remarkable applications of their tool will be presented in the Section 3.4.

Other groups have been working on similar approaches. For instance Thoma et al. studied the forward scattered $\mathrm{THz}$ signal from a SNOM tip for different time delays [52]. They show that the lateral recorded scans strongly depend on the time delay.

Emission scatterer Beside what has been described above, other configurations have been investigated. The Zhang's group in Troy also used a tip to scatter the $\mathrm{THz}$ field. But their generation of the $\mathrm{THz}$ was done directly beneath the tip on the semi-conductor under investigation, as presented in Fig. 10. They call this configuration a Near-field Emission Microscope [53]. They use an AC bias voltage between the tip and the semi-conductor to lock the detected signal. The dipole moments induced by the laser in the semiconductor materials are coupled to the tip and the bias causes an additional dipole moment at the sample surface. The recorded signal is strongly dependent on the tip to surface distance featuring a transition from no signal to full signal in a scale of $1 \mathrm{~nm}$. Using their THz-TDS system, they show a lateral resolution of $100 \mathrm{~nm}$ limited by the diameter of their tip.

\subsection{Guided mode on a wire}

Cylindrical wire Beside using the tip as a scatterer, one can try to use the tip to guide the wave and concentrate the field at its end. It has been proven that a $\mathrm{THz}$ field can propagate along a metallic wire via the radially polarized guided modes, called Sommerfeld waves which exist [54]. Adam et al. succeed to couple a field linearly polarized field to a metal wire, using a discontinuous phase element (DPE) as shown in Fig. 11a [55]. The radial mode propagates toward the wire end, and, due to its radial symmetry, it leads to a longitudinally 

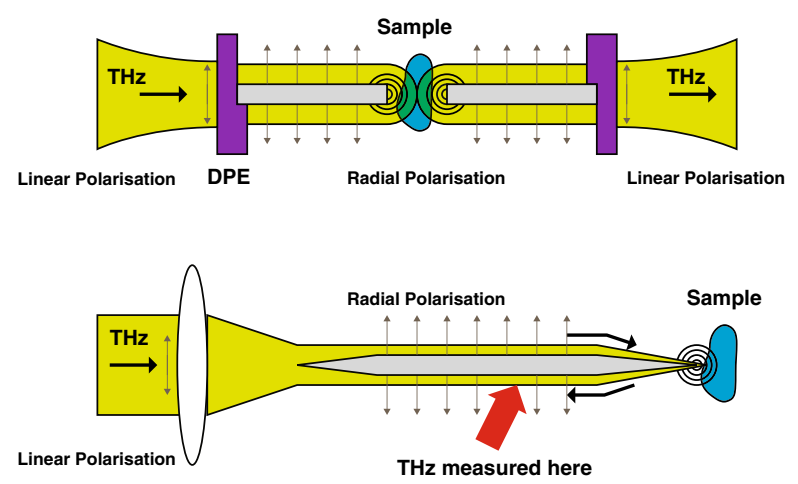

Fig. 11 Above The incident linearly polarised $\mathrm{THz}$ beam is coupled to a radial mode on a wire via a discontinuous phase element (DPE). At its extremity a strong field is present that could couple to an other wire [55]. Below The THz beam is coupled to the tapered wire. The tapering increases the strength of the field at the apex. The reflected guided mode is picked up by a EO probe along the wire on its way back [56].

polarized subwavelength confinement at the end of the apex. The apex can be used as a near-field source. Similarly, the apex can also collect any longitudinal field that will excite the radial mode of the wire, propagating towards the DPE. The guided mode will then be transformed into a linearly polarized freespace propagating beam that can be measured. This will constitute a passive probe antenna which selectively captures only the longitudinal component of the field. Using a $12 \mathrm{~cm}$ long tungsten wire of $0.6 \mathrm{~mm}$ diameter, the authors reached subwavelength resolution when measuring subwavelength holes. Unfortunately, the DPE can only work at a single frequency, in this case $100 \mathrm{GHz}$, which is at the limit of the THz domain. But they are confident to be able to demonstrate similar results at higher frequencies.

Tapered wire Meanwhile, Nagel's group in Aachen built a THz TDS setup based on a tapered wire as near-field probe [56]. They have tapered at both ends a $150 \mathrm{~mm}$ long, $1 \mathrm{~mm}$ diameter metallic wire. The taper angle was of the order of $10^{\circ}$ for a tip radius of $10 \mu \mathrm{m}$. To couple the incident $\mathrm{THz}$ wave, they focus it with a lens at the tip on one side. The radially polarized beam propagates along the wire and reaches the other tapered end as seen in Fig. 11. There the field is confined to dimensions smaller than the wavelength determined mainly by the tip diameter as shown experimentally in [57], leading to a possible superfocusing effect of the field $[58,59]$. Part of the pulse $(22 \%)$ is reflected back if the end of the wire is open. If a sample is placed at the vicinity of the tip of the wire, a strong reflection occurs and it contains information on the dielectric nature of the sample. In order to measure the $\mathrm{THz}$ pulses propagating along the wire, a $\mathrm{THz}$ switch detector is placed at the vicinity of the wire. Both the primary and the reflection pulses can be recorded. The authors could perform near-field measurement on a pair of $20 \mu \mathrm{m}$ metal lines separated by $80 \mu \mathrm{m}$, deposited on a semi-insulating GaAs substrate. The metal 
will reflect more the incident pulse than what will reflect the GaAs. This leads to a contrast that is stronger than the one obtained using the tip scattering method. A $20 \mu \mathrm{m}$ resolution at $0.3 \mathrm{THz}$ center frequency $(\lambda / 50)$ could be reached.

\subsection{Direct detection in the near-field}

As we've seen previously, to observe the near-field, one needs to bring in the near-field a filter (aperture) or a scatterer (tip). These two methods have the disadvantage to reduce the strength of the field measured. They also induced a significant distortion of the waveform. An other approach has been investigated, related to the source or to the detector size: thus if the source or the detector active size is smaller than the wavelength, and the source or the detector are placed in contact with the sample, the field collected will be strongly related to the area in contact with the source or the detector as seen in Fig. 12.

Source close to the object The group of Boccara in France has developed the idea to generate the $\mathrm{THz}$ radiation in the immediate vicinity of their sample [60]. In their case, they use a nonlinear crystal such as ZnTe or GaAs (500 $\mu \mathrm{m}$ thick) as local source and a bolometer for detection. The sample of study is placed directly on the emitter crystal. The femtosecond laser pulse is focused on the back of the emitter crystal, generating locally a THz pulse.

The size of their source is much smaller than the wavelength: it depends primarily on the focus spot size of the near-IR laser and the thickness of the emitter crystal. A resolution of $75 \mu \mathrm{m}$ at $1.5 \mathrm{THz}(\lambda / 3)$ was achieved. Using a deconvolution algorithm and a thinner ZnTe crystal $(200 \mu \mathrm{m})$, an improvement by a factor of two can be achieved [62]. The advantage of this technique is that it provides a signal completely independent of the sample topography in a rather simple configuration for the set-up.

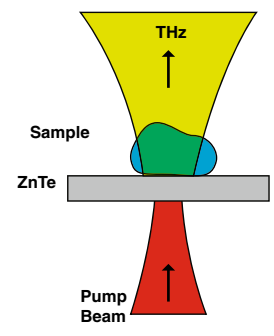

(a)

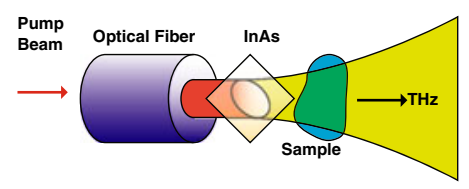

(b)

Fig. 12 Source in contact with the sample: a The THz radiation is generated by a pump beam focused on a ZnTe crystal. The sample is placed directly in contact with the crystal [60]. b The pump beam is coupled into a optical fiber. At the output of the fiber, a thin InAs crystal is placed, generating the $\mathrm{THz}$ beam. The sample adjoins it [61]. 
Recently, Yi et al. in Korea developed a tiny source using an InAs film coated on a $45^{\circ}$ wedged optical fiber tip [61]. At $0.5 \mathrm{THz}$, the authors obtained a $180 \mu \mathrm{m}$ spatial resolution $(\lambda / 3)$, equivalent to three times the size of the optical fiber core they have been using. The obvious advantage of the technique is the easy alignment by using the optical fiber as a compact $\mathrm{THz}$ emission tip.

Detector close to the object Similarly to the aperture case, bringing the detector directly in contact with the sample, in the near-field, would give an improvement of the resolution, provided the detector is small enough. When dealing with a TDS system, using electro-optical sampling could achieve this: the beam from the femtosecond laser, used to probe the change of index due to the $\mathrm{THz}$ radiation inside the crystal, has a radius much smaller than the $\mathrm{THz}$ beam wavelength because it is related to its own wavelength, typically $800 \mathrm{~nm}$. This probe beam can be used as a synthetic aperture: the detected $\mathrm{THz}$ radiation will be the one present on the path of the probe beam, as shown in Fig. 13.

As an early example, we can cite the work from Jepsen's group in Denmark [63]. They send the probe pulse into a ZnTe EO crystal from the back surface with respect to the direction of the incoming $\mathrm{THz}$ pulse. They measure the near-field of their emitter, a bias GaAs antenna. In this example they were not aiming at a tiny focus of their probe beam. The resolution achieved with their method was $0.5 \mathrm{~mm}$. In their configuration, they were only interested in the main components of the field, i.e. the propagating component emitted by their source.

Whitaker's group shows a similar method at microwave frequencies (100 $\mathrm{GHz}$ ) [64]. They measure the radiated field from a microstrip patch antenna

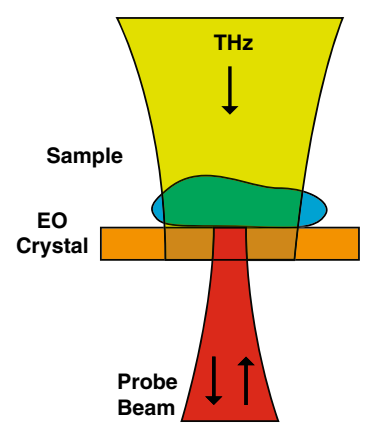

(a)

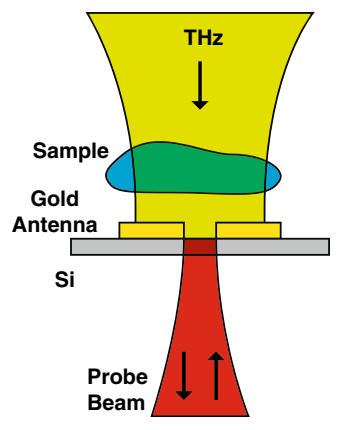

(b)

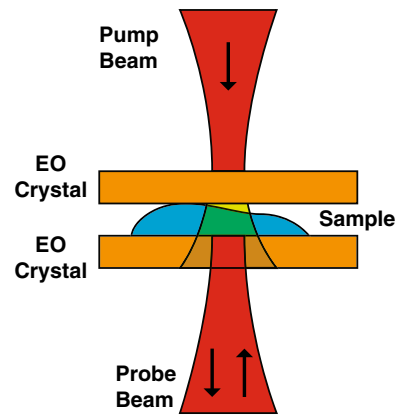

(c)

Fig. 13 a The THz radiation is focused on the object, placed on top of an EO crystal. A counterpropagating probe beam will measure the different components of the THz electric near-field [67]. b Instead of the EO crystal, a photoconductive $\mathrm{THz}$ detector is used [10, 69]. c Quasi near-field approach: the sample is placed between two EO crystals, one to generate the $\mathrm{THz}$ beam, one to measure it [70]. 
comparing GaAs, bismuth silicate (BSO) and lithium tantalate $\left(\mathrm{LiTaO}_{3}\right)$ probes. Because they use different crystals as probes, they are able to measure independently the different components of the field in a plane above their antenna. They obtain two-dimensional maps of the electric-field amplitudes and phases with a spatial resolution of $8 \mu \mathrm{m}(\lambda / 375)$. Seitz et al. implement the same configuration for larger bandwidth and higher frequencies [65]. They use an external EO sampling based on a $\mathrm{LiTaO}_{3}$ crystal to measure a $\mathrm{THz}$ pulse that propagates along a coplanar stripline on GaAs. As in the first case, the probe made of EO crystal was moved above the sample. Because the distance between the probe and the sample influence your measurement, the authors already advice to work with a distance sample/tip of $0.5 \mu \mathrm{m}$.

One solution to overcome this issue is to design a probe which minimizes its interaction with the sample or make it more uniform. This strategy has been followed by Planken's team in collaboration with Dai Sik's group. The tip is replaced by a large EO crystal $(\mathrm{GaP})$ placed in contact with the sample. The polarisation of the counter-propagating pulse and the orientation of the crystal are such that one can measure independently all the three components of the electric near-field [66]. The first measurements they've presented were on the diffracted field from a single slit and from an one-dimensional slit array [67]. A $\mathrm{THz}$ beam is focused on one side of the sample. A large EO crystal is in nearcontact with the sample on the other side, with an air-gap of about $50 \mu \mathrm{m}$ as described in Fig. 13a. The counter-propagating probe laser pulse is focused to a small spot less than $10 \mu \mathrm{m}$ away from the front surface of the EO-crystal, using a reflective objective. It is then reflected from the front surface. A reflection coating made of $\mathrm{SiO}_{2}$ is deposited at the front surface in order to reflect the probe laser pulse. A layer of Ge is deposited on top in order to avoid any leakage of light coming from the probe beam. Once reflected, the probe beam is sent to a standard differential detection setup.

The presence of a $\mathrm{THz}$ field inside the EO crystal will change the refractiveindex and affects the ellipticity of a circularly polarized probe beam. The EO crystal is raster-scanned in all directions $(x-y-z)$ to measure the temporal evolution of the electric field. Three different configurations can be set in order to record the three different components. In [67], they have measured the $\mathrm{THz}$ radiation emerging from a slit made in a metal foil with subwavelength dimension $(50 \mu \mathrm{m})$. Because of the symmetry of the system, only measuring the $x$ and the $z$-component in the $x-y$ plane is relevant. They show the propagation of the wave front for both components with a resolution of $20 \mu \mathrm{m}$ and a bandwidth of $1.5 \mathrm{THz}$. They illustrate their technique by measuring the near-field of a metal sphere and of a single square hole in a metal foil [68].

The near-field component perpendicular to the structure is the most difficult to measure. Its amplitude decays rapidly when the distance sample-crystal increases. In order to have a more accurate measurement of this component, the authors have decided to directly deposit the structure on the EO crystal. They can only measure the electric near-field in one plane, but they avoid the presence of a gap that would influence the measurement. They've measured 
the full $\mathrm{THz}$ electric near-field emerging from a single aperture deposited on the EO crystal [9].

To obtain high resolution using an EO crystal, the electro-optic interaction region has to be relatively small: the probe beam should be well focused and the EO crystal has to be thin. But this will reduce the change of ellipticity of the probe beam the detected signal. To overcome this effect, Mitrofanov proposes to enhance the electro-optic effect in the thin EO crystal (typically $3 \mu \mathrm{m}$ ) via optical resonators [71]. The photon from the probe beam is trapped inside the active cavity where the electro-optic phase shift occurs by two distributed Bragg reflectors. The electro-optic effect can be enhanced leading to a spatial resolution of less than $1 \mu \mathrm{m}$ for operating frequencies from 0 to $3 \mathrm{THz}$. The bandwidth detected is strongly related to the duration of the probing optical pulse inside the cavity; increasing the $Q$-factor of the resonator will give rise to a stronger electro-optic signal but it reduces the bandwidth.

One draw back of such a method based on a focused probe beam is the low speed for acquiring the image: the sample is mechanically raster scanned pixel by pixel. To improve the speed, Tanaka's group has combined the EO sampling with a charge-coupled-device (CCD) camera [11]. The probe beam is collimated with a diameter of $2 \mathrm{~mm}$ on the back of a ZnTe crystal used as $\mathrm{EO}$ crystal. The incident $\mathrm{THz}$ beam is focused on the front of the crystal with a similar diameter so the probe beam covers entirely it. The probe beam is then reflected by the front side of the crystal and propagates colinearly with the $\mathrm{THz}$ beam inside the crystal. As for the case of a focused probe, the polarisation is modulated by the $\mathrm{THz}$ electric field. An analyzing polarizer converts this modulation into an intensity modulation which can be detected further on by the $512 \times 512$ pixels CDD camera. The authors show how they can visualize the electric near-field over a large area at a 2.7 frames per second. Unfortunately, the spatial resolution determined by the camera and the optics is more coarse then the one obtained for a focused probe beam. Nevertheless, they've reached $70 \mu \mathrm{m}$ resolution for a peak intensity at $300 \mu \mathrm{m}(\lambda / 4)$.

An other probe, not based on an EO crystal, has been introduced by the Walther's group in Freiburg [69]. They operate a TDS system with a siliconon-sapphire detector chip instead of an EO crystal as near-field probe see Fig. 13b. An H-shaped electrode is deposited on the silicon wafer and the probe laser beam is focused through the sapphire substrate into the $10 \mu \mathrm{m}$ wide photoconductive gap which defines together with the probe beam focus, the maximum resolution at $20 \mu \mathrm{m}$ at a frequency of $0.7 \mathrm{THz}(\lambda / 20)$. The measurement is sensitive to the in-plane polarisation of the $\mathrm{THz}$ near-electric field but can not be done in contact with the sample: the distance between detector and sample is experimentally limited to $35 \mu \mathrm{m}$.

The group of Nagel in Germany has brought the latter concept to a commercial product http://www.amo.de/?id=687. It manufactures a freely-positionable photoconductive probe-tip mounted on a wedge with a $30^{\circ}$ inclination angle. The design of the electrodes deposited on low-temperature grown GaAs features two tapered planar strips. The gap between them at the apex is about $1.8 \mu \mathrm{m}$ wide. A photograph of the probe is presented in Fig. 14 with a schematic 
(a)

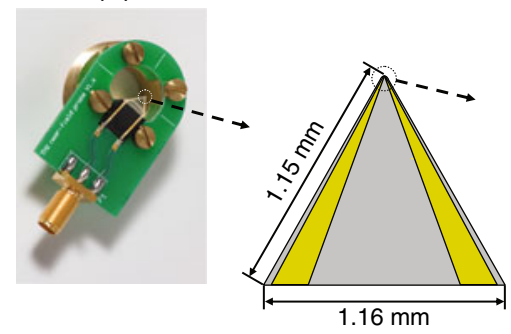

(c)

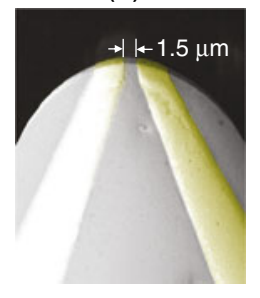

Fig. 14 a Photograph of the tapered photoconductive probe mounted on a wedge with a $30^{\circ}$ inclination. b Schematic of the probe tip. The underlying LT-GaAs is colored lime and the tapered wires are colored yellow. $\mathbf{c}$ Zoom image into the tip region with a slightly reduced gap size $(1.5 \mu \mathrm{m})$ than presented in [10].

of the tip and a zoomed picture of it. More details on the probe can be found in [10]. Due to the electrode configuration, the probe is sensitive to both in-plane components of the electric near-field. But the sensitivity difference between both polarization presents a 11:1 ratio, the probe being more sensitive to the polarization parallel to the gap than the perpendicular one. They achieve a $5 \mu \mathrm{m}$ spatial resolution for a bandwidth of $0.1-3 \mathrm{THz}(\lambda / 600)$ for the main component, $7 \mu \mathrm{m}$ for other. Results obtained by these methods will be further discussed in the Section 3.1.

Quasi near-field The idea to bring either the source or the detector in the near-field led naturally to the combination of both method in a so called "quasi near-field" set-up [70]. The authors use EO crystals for generation and detection close to each other, with the sample sandwich in between. Because of the proximity between the source and the detector, the signal-tonoise ratio (SNR) was very high, and no dry nitrogen flushing was required to remove water vapor absorption from the spectra. Unfortunately, the actual configuration doesn't enable imaging: both crystals are clamped and adjusted with respect to the generation and probe beams and they can not be moved, see Fig. 13c.

\section{Applications}

The techniques which have been developed in the $\mathrm{THz}$ domain to reach subwavelength resolution images of the near-field have created and inspired many new exciting studies. We will try to give an overview of the most inspiring research achieved so far. We can classify the different inquiries as follow: first we will focus on the fundamental studies, mainly based on theoretical physics. Then, we will introduce examples of inspection-like applications and spectroscopic analysis at micro-scale. Finally, we will present applications outside physics, for instance in biology. 


\subsection{Apertures}

One of the main advantage of the THz-near-field TDS techniques versus optical near-field ones is the possibility to measure the electric field, both in amplitude and in phase. Certain of these techniques can even measure the total vector field, i.e. the three components of the electric near-field. Undoubtly these techniques can be of great help when studying the behavior or light where knowledge of the direction of the field vector is important.

Circular holes The paper that brought the near-field in the spotlight was certainly the paper by Ebbessen et al., published in the late 90s [1]. In this paper, the authors report on the extraordinary optical transmission through subwavelength hole arrays. Such behavior of "light" has already been observed for the microwave region [72] and even, recently, in the THz domain [73]. But the reason would be different for the optical wavelength, due to a coupling of light with plasmons at the surface of such periodic structure. Therefore, a great rush arose to measure the field at the vicinity of these sub-wavelength structures.

Before studying the complication of a periodic system, one has to address the diffraction of light by a isolated hole. Already in the 40's, Bethe and Bouwkamp analytically studied the light propagation through a punched hole on an infinitely thin perfect conductor. Remarkable results like the fact that the hole differentiates the incident field, waited to be measured. In 2008, Adam et al., using an EO crystal underneath a hole made of a deposited gold layer, compared successfully the Bouwkamp's model with the measurement of the $\mathrm{THz}$ light emerging from the subwavelength hole [9]. They could especially measure the component perpendicular to the metal sheet, i.e. the main nearfield component. They gave the distribution of the phase underneath the hole for this component. In a following paper, they measured the other components of the $\mathrm{THz}$ electric near-field [74]. The results can be seen in Fig. 15, with the three components of the electric near-field at $0.2 \mathrm{THz}$ for a $150 \mu \mathrm{m}$ diameter hole.
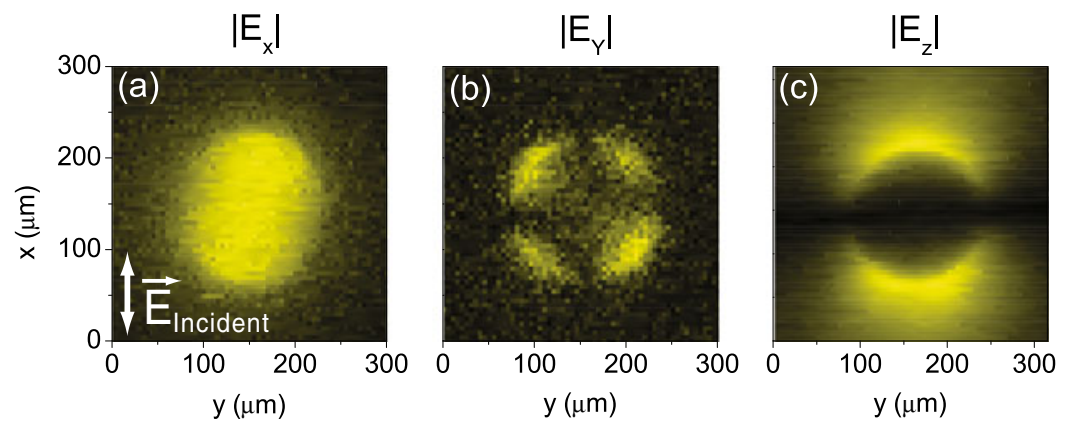

Fig. $15 \mathrm{THz}$ electric near-field for a single, isolated, circular aperture $(d=150 \mu \mathrm{m})$ at $0.2 \mathrm{THz}$. $\mathbf{a}-\mathbf{c}\left|E_{x},\right| E_{y} \mid$, and $\left|E_{z}\right|$. The scan area is $315 \mu \mathrm{m} \times 315 \mu \mathrm{m}$ [74]. 
The Delft group continued its research of isolated apertures and studied the effect of the presence of a substrate underneath the hole [75]. They could compare measurements for different thickness of metal foils with numerical simulation. They found out that for very thin metal layers, the hole acts as if it were filled by the substrate, and therefore exhibits a much lower cut-off frequency than expected by the waveguide theory.

Using the antenna-detector approach, Walther's group was able to observe the interaction of the $\mathrm{THz}$ light emerging from various circular subwavelength holes by measuring the in-plane component of the THz electric near-field [69]. They could differentiate between the propagating and stationary modes occurring after the diffraction at the holes. They could image standing wave patterns on the metal surface between successive holes. This work is fundamental to understand the mechanisms for the extraordinary transmission of light through periodic hole arrays.

Similar standing waves can be found when exciting a microwire with a incident $\mathrm{THz}$ beam [76]. Constructive wave interferences and the formation of standing wave patterns occur due to the counter-propagating waves created at both ends of the micro-wire. We will further discuss the wire measurements in a later paragraph.

Nano-apertures Beside circular holes, other apertures may be of interest. Rectangular apertures, for instance, present remarkable properties. GarcìaVidal et al. have shown numerically that rectangular apertures exhibit transmission resonances near their cut-off wavelength [77]. Furthermore, they've predicted that a large enhancement of the electric field at the hole can be expected. Near-field measurements of rectangular holes have been performed by Seo et al. with $\lambda / 100$ resolution [78]. They proved that the near-field amplitude becomes proportionally larger as the rectangle becomes narrower as expected by the simulations. A large field enhancement at the resonance is also observed. These results suggest that a further decrease of the width of the rectangular hole will increase even further the near-electric field. Measurement in the far-field has been conducted with a 70-nm width nanogap perforated on gold film [79]. It shows that $\mathrm{THz}$ light can leak through such aperture, despite the ratio wavelength-aperture gap equivalent to $\lambda / 30,000$. This could lead to applications for super-focusing devices or ultra-broadband filters at $\mathrm{THz}$ frequencies [80].

\subsection{Theoretical study}

Beam profiling The subwavelength resolution enabled by the existing techniques can even be used to study simple cases of light propagation, for example that of focused field. Already in 2000, Gürtler et al. have studied the pulses emitted from large-aperture (LA) antennas in both the near-field and the farfield [63]. But they used conventional techniques, with a resolution limited to the size of the probing beam inside their EO detection crystal, i.e. $0.5 \mathrm{~mm}$. Similar measurements of the $\mathrm{THz}$ field pattern in focus have been done by 
Bitzer et al. with the identical limitation [81]. An improvement of spatial resolution has been achieved by the same group, using a photogated gap detector. They reached $40 \mu \mathrm{m}$ spatial resolution [82]. They could measure the field distribution in the focal plane of the silicon lens and observe that the diffraction effect at the lens aperture influences the spectral beam profile in focus.

More interesting beam profiles can be studied using this technique. Radially polarized beams for example are known for their smaller beam waist and their strong longitudinal field components in the focal plane. Winnerl et al. developed new microstructured photoconductive antennas capable of producing radial and azimuthal polarization [83]. They have measured the beam profiles of divergent and refocused beams emitted by these antennas. They could show that these can be described as lowest-order Bessel-Gauss beams. The beams could be used to couple more efficiently to guided $\mathrm{THz}$ modes, for example present on wires.

Magnetic field Because certain of these techniques give an accurate value of the components of the electric field (three for the Delft group, and two in plane for the Freiburg group for example), one can use it to calculate, via Maxwell's equations, the magnetic near-field component perpendicular to this plane. Seo et al. first applied this method to the near-field beneath periodic slits made from a thick metal plate [67]. The incident polarization is along the $x$-axis, perpendicular to the direction of the slit. Considering the slit infinitely long along the $y$-axis, the problem can be reduced to a $2 \mathrm{D}$ problem in the plane $x-z$. They've measured the $E_{x}$ and $E_{z}$ components in the plane $x-z$ underneath the slits. They could extract the magnetic near-field $H_{y}$ at the plane and also calculate the Poynting vector $\mathbf{S}$ in that plane. This last vector gives the direction of energy propagation after diffraction by the slits. In Fig. 16, the magnetic field profile at $1 \mathrm{THz}$ is plotted, together with the electric field vectors.

Metamaterials The Freibourg group took a similar approach for a measurement on a metamaterial structure [84]. These structures exhibit specific resonances in transmission when measured in the far-field. These properties can lead to extraordinary behavior such as cloaking [85]. For an overview of the field of metamaterials, see [86].

For their measurement, Bitzer et al. measured the in-plane electric nearfield beneath a split-ring resonators (SRR). Inside the two rings of their SRR, circulating currents are generated due to the incident $\mathrm{THz}$ field which couples to them via their openings. The circuit can be seen as an LC circuit producing resonances. Thanks to these currents, there is emergence of a magnetic moment normal to the SRR plane inside the rings. By calculating the magnetic field via their measurement of the electric near-field, they could image each fundamental mode that is responsible for the resonance in the farfield. Via the calculated magnetic field, they could predict the current density in each of the arms of the structure and compare it with current densities given by FTDT simulation. The near-field distributions of a single SRR are 


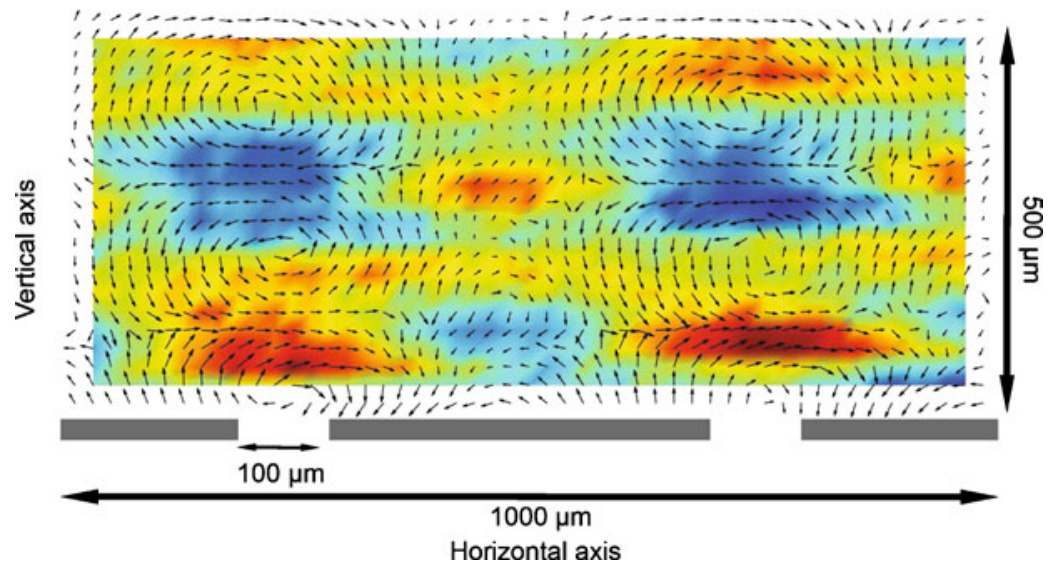

Fig. 16 Measured light emanating from a multiple slit sample of $100 \mu \mathrm{m}$ diameter with a periodicity of $500 \mu \mathrm{m}$ at $1 \mathrm{THz}$ : the black line denotes the electric field and the background color represents the magnetic field. The fields is chosen at an fixed phase within one oscillation cycle [67].

shown in Fig. 17, taken from [84] for four different resonances. The two higher resonances, given in at $\mathrm{A} 3$ and $\mathrm{A} 4$ present an electric quadrupole excitation while the two lower show a rotational field oscillating around the center of the structure.

(a)

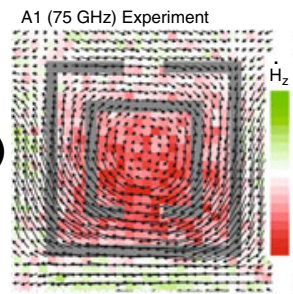

A2 $(150 \mathrm{GHz})$

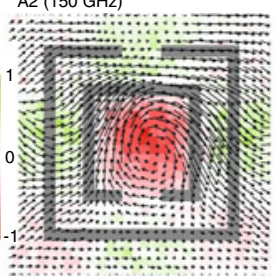

A1 $(80 \mathrm{GHz})$ Simulation

(b)

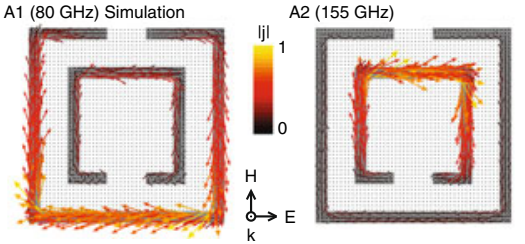

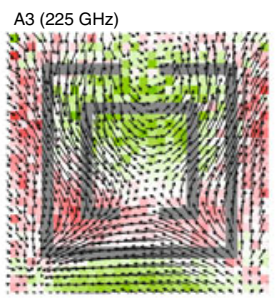

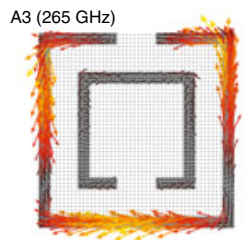

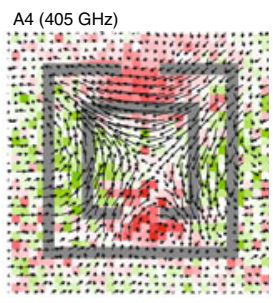

A4 $(450 \mathrm{GHz})$

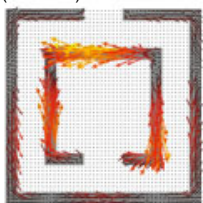

Fig. 17 a Measured electromagnetic near-field distribution and simulation of the current density. The vector plots show the electric in-plane field vectors in the $x$-y plane at the backside of the sample at different resonances for an isolated SRR sample. The color code indicates the time derivative of the out-of-plane component of the corresponding magnetic field, derived from the electric field vectors. The field is chosen at a fixed phase within one oscillation cycle. b Simulated surface current density. Polarization and propagation direction of the incident wave is indicated in b [84]. 
Furthermore, the same team revisited Babinet's principle by studying complementary planar metamaterials in the near-field [87]. One should expect the scattered electric and magnetic fields to be complementary for complementary structures. To demonstrate this, they've measured the electric near-field of a split-ring resonator and its complementary version. They calculated the magnetic field perpendicular to the plane of measurement of the in-plane electric near-field, for a resonant structure with a linearly polarization parallel to the SRR gap. They compared the result with the in-plane electric nearfield of the same structure with a linearly polarization perpendicular to the SRR gap. They found a good agreement between the two measurements, as predicted by Babinet's principle. Some minor issues rise from the fact that the measurement is not done in contact with the structure and, therefore, the field lines are not perfectly perpendicular to the metal surface as expected for a perfect conductor.

It has to be noted that measuring directly the magnetic near-field is very challenging. At $\mathrm{THz}$ frequencies, only the transient magnetic component of a freely propagating $\mathrm{THz}$ beam has been measured using the Faraday effect in a magneto-optical crystal [88]. At optical wavelengths, only recently some indirect measurements have been performed [89]. It shows that calculating the magnetic near-field via the measurement of the electric near-field is up to now the best way to understand the mechanism of resonances in metamaterials.

Acuna et al. did perform measurements on planar $\mathrm{THz}$ resonators, using their tungsten probe to scatter the near-field [90]. They were able to study in the near-field the high frequency resonances of these metamaterials.

Wachter et al. proved that they could use their PC antenna probe shown in Fig. 14a to measure the electric near-field in the vicinity of an asymmetric double-split metallic ring resonator with subwavelength dimension, presented in Fig. 18a [10]. Figure 18b gives the THz peak amplitude at a given moment in time of the electric near-field in a plane under the structure. Both the metallic strip and the gaps are clearly resolved in the image.

Fig. 18 a Layout of a single element of the frequency-selective surface (FSS) used for experimental characterization. Dimensions are given in $\mu \mathrm{m}$; $\mathbf{b} \mathrm{THz}$ near-field image of the FSS. The image consists of $50 \times 50$ pixels with a $3 \times 3 \mu \mathrm{m}^{2}$ pixel size [10].
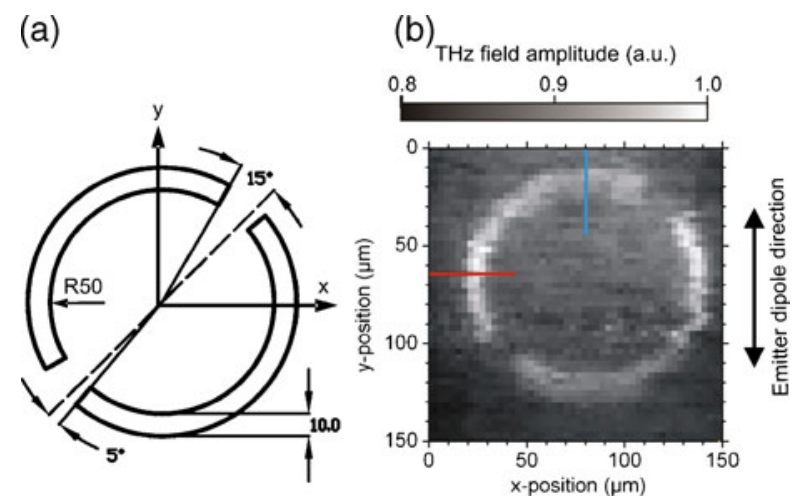
This last example shows that $\mathrm{THz}$ near-field imaging is now relatively accessible to anyone using a commercially available tip.

\subsection{Plasmonics on a flat surface}

Over the past two decades, most of the attention in optics has been dedicated to plasmonics, i.e. the study of collective oscillations of a free electron gas. Surface plasmons (SPs) are very exciting plasmonic waves because they enable the light to propagate at the interface between any two materials with specific properties, like air and gold for the visible wavelengths. SPs are associated to other electromagnetic propagation modes on surface like the Zenneck waves on a flat metal surface [91] and the Sommerfeld wave on a single metal wire [92]. These last two types of surface waves have been measured at $\mathrm{THz}$ frequencies [54, 93]. These surface waves can be used, for example, to perform $\mathrm{THz}$ thin film spectroscopy $[94,95]$. Because metals can be considered as almost perfect conductors at $\mathrm{THz}$ frequencies, it is difficult to distinguish between SPs and Zenneck waves. This leads to heated discussions between the $\mathrm{THz}$ and the optical surface plasmon communities on the existence of SPs at $\mathrm{THz}$ frequencies. Near-field measurements would without ambiguity answer this question.

Nahata's group at the University of Utah has been working intensively on the corrugated hole described in Section 2.4. The annular grooves couple the incident wave into surface waves that contribute to the increase of transmission through the hole. Via electro-optic sampling at the vicinity of the surface, the researchers succeeded in measuring the electric near-field vector associated with the THz surface waves [96]. Using the same apparatus, they've measured the Gouy phase shift at the focus of surface waves created by a semi-circular groove on a metal foil [97]. It is clear that these techniques are suitable for measuring any surface wave on flat surfaces. One can think to study particular planar structures such as described by Pendry which would mimic SP properties at $\mathrm{THz}$ frequencies [98]. To get closer to what is happening in the optics range, one can propagate $\mathrm{SP}$ at $\mathrm{THz}$ frequencies on surface gratings structured on doped silicon surfaces [99]. Studying these in the near-field would give a good insight in SPs.

Plasmonics on wire Besides on a flat surface, these surface waves can propagate as Sommerfeld waves on a wire. These waves are extensively used in $\mathrm{THz}$ near-field imaging as shown in Section 2.6. The Aachen group directly studied the field at the wire itself using their PC probe-tip based setup [100] which leads to use tapered wire as near-field probe [10]. The Mittleman group has measured the spatial profile of the electric near-field at the end of a wire where $\mathrm{THz}$ waves were propagating. The measured profiles of one component of the electric near-field are reproduced in Fig. 19 [54]. One can clearly see the radial nature of the waves in this example.

Astley et al. pursued the study of the confinement of the $\mathrm{THz}$ field at the end of the metal wire. The authors map the field at the end of two different 


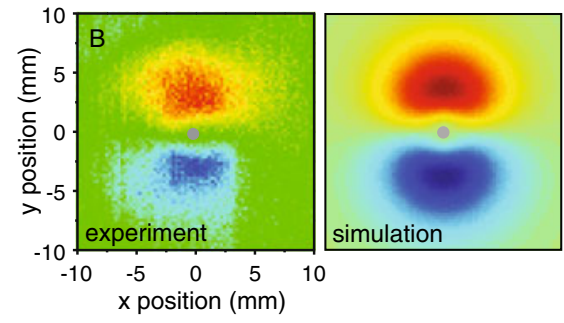

(a)

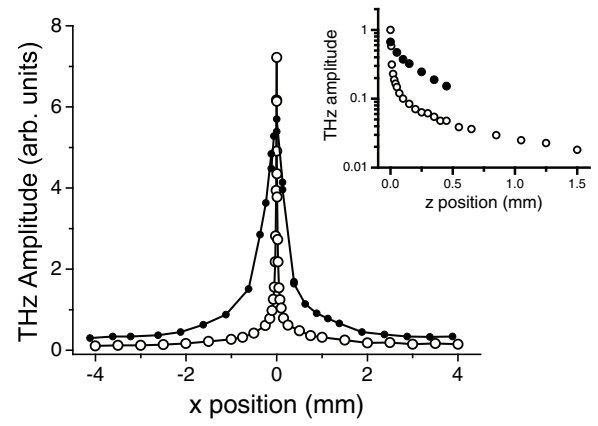

(b)

Fig. 19 a Measured spatial profile of the electric near-field [54]. b Simulation of the vertical component of the electric field for a cylindrically symmetric radial mode [101].

wire waveguides, one with a flat end, and the second with a tapered end [101]. Figure 19 shows the $z$-component of the electric near-field, parallel to the wire, at its apex along the $x$-direction, perpendicular to the wire axis. A sharp probe was used to scatter the field in combination with a $\mathrm{THz}$ receiver, placed $90^{\circ}$ out of plane. The two cases (untapered and tapered) show different field distributions with a full width at half maximum (FWHM) of $630 \mu \mathrm{m}$ for the untapered wire and $30 \mu \mathrm{m}$ for the tapered one size, a comparable to the tip size. This suggests a very strong sub-wavelength confinement of the $\mathrm{THz}$ electric field.

Likewise, Adam et al., in France, measured the longitudinal component of the electric near-field propagating along a wire using a similar tapered wire as a probe [55]. They could even show the coupling between two adjacent wires, one used as a carrier for the $\mathrm{THz}$ waves, the other one used as a probe.

Evidently, more near-field measurements of these radial modes will be performed in the future as new ideas emerge. A good illustration would be the corrugated wire presented by Maier et al. [102].

Waveguide The near-field imaging technique is also a great tool to study waveguides, especially the mode that can propagate in it. Mitrofanov et al. made a worthy use of their near-field set-up to identify the mode structure and composition in dielectric-lined hollow metallic waveguides [103]. They could match the measured spatial profiles, both in amplitude and phase, over a large frequency band with the expected modes for such waveguide. Information on the mode group velocity dispersion can also be extracted from these measurements.

Fibers can also be studied using near-field imaging. Thus, the modes of a polymer photonic crystal fiber have been characterized by Nielsen et al. [104]. The authors prove that the structure was single-moded for a wide frequency range. Higher modes were also present for higher frequencies. They've shown that the solid core of their photonic crystal structure acts as the main guiding 


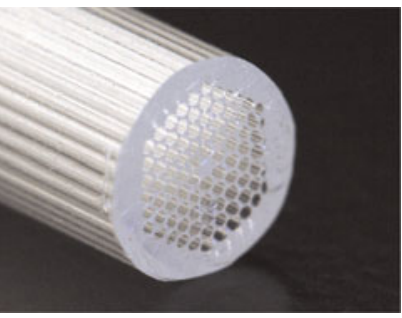

(a)

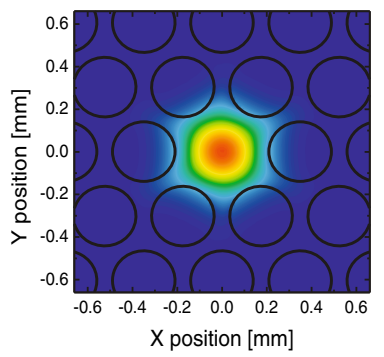

(b)

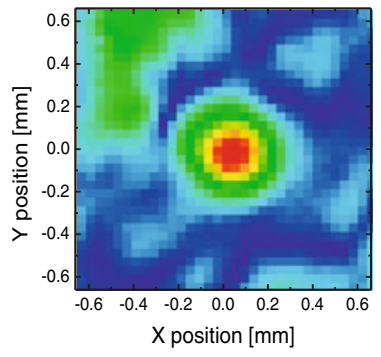

(c)

Fig. 20 a Photograph of the small mode area (SMA) fiber. b Calculated fundamental mode structure at $1 \mathrm{THz}$ for the SMA fiber. c Measured modal structure of the electric field of the propagating mode through the SMA fiber [104].

element. Profiles of the electric near-field at the end of the fiber are shown in Fig. 20 with a photograph of the fiber itself.

These results shows how enlightening $\mathrm{THz}$ near-field imaging is by offering both amplitude and phase information on the mode distribution inside waveguides or fibers. It has to be stated that no similar tool is available for optical nor near-infrared fibers. Interferometry solutions in the visible exist but can't measure independently all components of the electric field [105].

One can foresee the use of this technique to study other types of planar waveguides such as Goubeau lines or the one recently suggested by MarinCano et al. based on domino-like chains placed on metallic surfaces [106].

\subsection{Inspection}

Most of the experiments performed with the different $\mathrm{THz}$ near-field techniques based on the tip-scattering method described above have been applied to the inspection of surfaces and the study of microscopic properties of matter. To understand how these measurements work, we would first like to introduce the literature that explains the principle of the tip/sample interaction and then give some examples to illustrate the potential of the technique.

Tip/Sample interaction To grasp the reason of the strong beam-tip-sample interaction and the resulting subwavelength resolution, a good model had to be built. The first idea is to consider the tip as a simple Mie-scatterer and the sample as a planar surface. Using a quasi-electrostatic theory, Knoll and Keillman describe the interaction between a polarizable sphere (the tip) and its image inside the sample induced by the surface charges in the sample [39]. They show that the strong coupling between the tip and its image in the sample causes a steep increase of scattering cross-sections when the tip/sample distance is very small. They describe it as a non-linear behavior: the scattered signal contains many harmonics of the fundamental modulation frequency $\Omega$ of the tip. Because this effect is only valid when the tip is really close 
to the sample, only the part of the tip close to the sample will contribute to these harmonics. By filtering only these high harmonics, one can achieve background-free measurement.

To see the tip as a simple Mie-scatterer is a very candid way to model the tip. In 2004, Mittleman and Planken realized that in their tip set-up, the measured near-field is different from the incident field and somehow resembles the timeintegral of the incident field, an effect that the Mie-scattering can not predict [107]. To explain this observation, they do not consider the tip as a scatterer but as a dipole antenna, resistively and inductively coupled to the incident $\mathrm{THz}$ radiation. Treating the tip as a wire antenna can explain the bandwidth reduction observed in the near- and far-field configuration. The same approach has been discussed in [108] to explain the relatively slow decay of the near-field amplitude when the tip-crystal separation increases. It also shows that when the tip is modulated only the end of the tip is contributing to the observed signal and not the entire antenna. The sphere model can also be seen as a special case of the antenna model when the antenna length is small. Chen et al. have introduced a model where the tip/surface is considered as a resonant RLC circuit, similar to the one of [107]. They've deduced that this resonant electrical coupling between the tip and the surface is responsible for the nanometer spatial resolution of the $\mathrm{THz}$ near-field measurement [109]. Cvitkovic et al. present similar study, comparing the different models to each others [110].

The most important aspect of all these models is to understand the influence of the sample on the measured signal. It is clear that the generated image sphere or dipole will depend on the electrical properties of the sample i.e. the complex dielectric constant. When the sample is a metal, such as gold, the image can be considered as a mirror image; when the sample is a dielectric, such as silicon, the image will strongly depend on the electric properties. Therefore the scattered field will contain the information on the dielectric properties of the sample. In the right plot of Fig. 21, Ribbeck et al. give an example of the calculation of amplitude contrasts of the field scattered from a tip above a Si surface with different doping concentration [51]. One can see that at a fixed frequency, such as $1 \mathrm{THz}$, a strong contrast is expected between doping concentrations in the range of $10^{17}-10^{19} \mathrm{~cm}^{-3}$.

The Mittleman group performed an interesting study on the background signal of these small metal tips when illuminated by a THz pulse [111]. The modulation of the probe/sample distance is responsible of this additional signal to the classical signal, expected from the general approach of ANSOM measurement. Nevertheless, due to this effect, they show that these tips can also be used for scattering-probe imaging of freely propagating $\mathrm{THz}$ fields.

Carrier concentration With the results from numerical calculations above, one could expect to measure a contrast when the tip is above materials with different carrier concentration, and, therefore, map the carrier concentration of the semi-conductor. Earlier results from Ribbeck et al. have demonstrated this. The right plot of Fig. 21 gives the experimental THz s-SNOM amplitudecontrast spectra of two types of doped silicon, normalized to that of gold. 

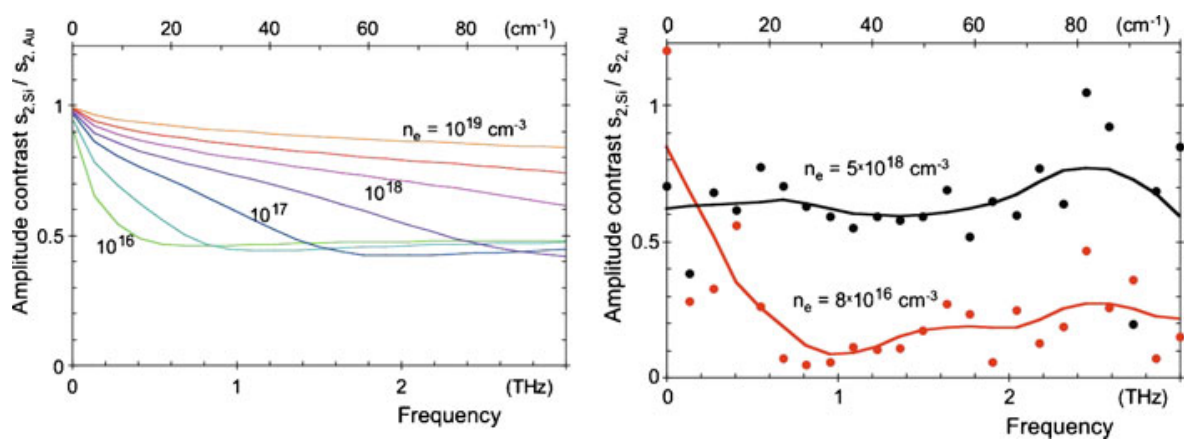

Fig. 21 Left Experimental THz s-SNOM amplitude-contrast spectra of doped Si, normalized to $\mathrm{Au}$ (experimental dots; the dashed curves are guides to the eye). Right Theoretical THz s-SNOM amplitude-contrast spectra of doped Si normalized to Au, calculated by the point-dipole/mirrordipole model for several free electron densities (right) [51].

The plot indicates a clear contrast between the two different concentrations. Following this initial measurement, Huber et al. could give a $\mathrm{THz}$ near-field image of a multiple-transistor device structure, reproduced in Fig. 22 [8]. The $\mathrm{THz}$ signal reveals the different mobile carrier concentrations within the $\mathrm{Si}$ substrate of the transistor, in much more detailed way than the same IR near-field image. Also the resolution achieved with their THz SNOM method $(\lambda / 3000)$ is, to this date, the smallest ever reported and enables a perfect image rendering of the carrier concentrations. The volume probed by the $\mathrm{THz}$ nearfield is reduced to a tiny volume, of the order of $40 \mathrm{~nm}^{3}$ which means that
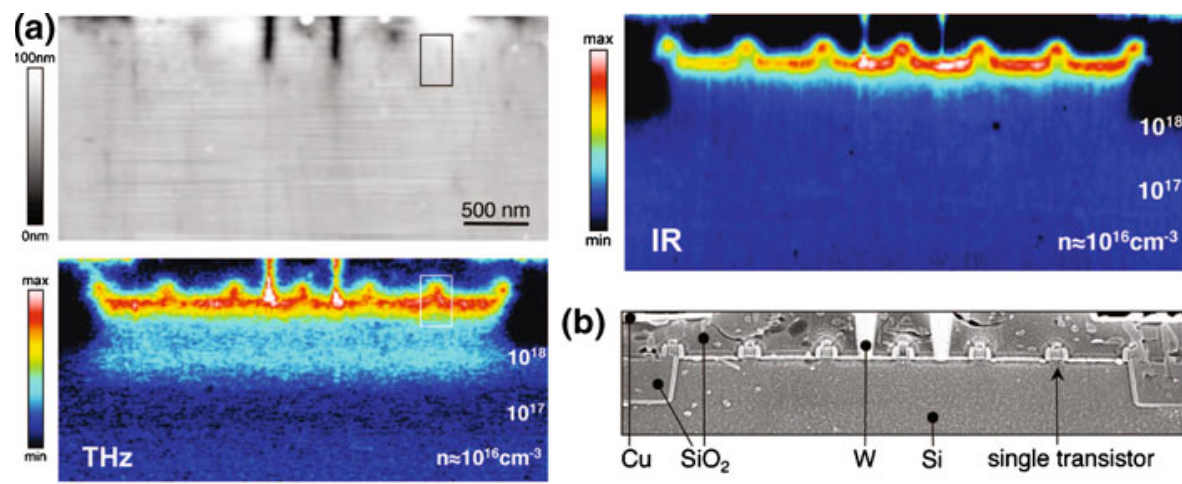

(b)

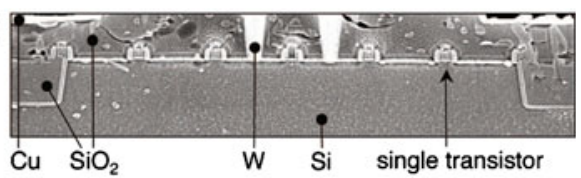

Fig. $22 \mathrm{THz}$ near-field microscopy of a polished cut through a multiple-transistor device structure. a AFM topography (gray, top-left) and simultaneously acquired THz near-field image (bottomleft). The varying $\mathrm{THz}$ signal within the Si substrate reveals the different mobile carrier concentrations $\mathrm{n}$ indicated by numbers obtained from device simulations. The infrared near-field image (top-right) $(\lambda=11 \mu \mathrm{m}$; taken for comparison) clearly demonstrates that only with $\mathrm{THz}$ illumination the varying mobile carrier concentration can be recognized. b The SEM image of a similar but decoration-etched sample validates that the $\mathrm{THz}$ image distinguishes different materials and the single transistors [8]. 
only few electrons are contributing to the $\mathrm{THz}$ response. This shows the high sensitivity of the method presented here.

Kersting group in Munich focused its research on the measurement of carrier concentrations. They used the same tip scattering approach but with an additional module, called electromodulation. The tip and the sample are electrically connected via an ohmic contact and an alternating bias will change the charge carrier density under the tip via the Schottky contact created. The depletion zone at the semiconductor surface is modulated via the bias and depends on the doping density of the semiconductor. The resulting modulated $\mathrm{THz}$ signal carries information only from the mobile charges and does not contain any contributions due to the semiconductor itself. A direct measurement of the permittivity can be performed this way $[112,113]$.

As an illustration, we give in Fig. 23a the THz image that Kersting group obtained from a two-dimensional semiconductor structure. Here, gold lines and $\mathrm{SiO}_{2}$ lines are deposited respectively vertically and horizontally on a semi-insulating silicon substrate. The different dielectric permittivities of the materials can be distinguished easily with a $1 \mu \mathrm{m}$ spatial resolution. In such a case, geometrical properties of the tip-surface can influence the $\mathrm{THz}$ signal. They are discussed in details in [48] as well as the description of the tip/sample interaction. Because they use a TDS system, spectral amplitude distributions of the modulated $\mathrm{THz}$ signal can be recorded, see Fig. 23b. In this case, the amplitude spectrum of the incident $\mathrm{THz}$ beam (dotted line) is compared to the measured modulated $\mathrm{THz}$ signal from a bulk n-doped GaAs structure (dots). It shows that the electronic response is dominated by low frequencies and can be understood via a simple RLC model.

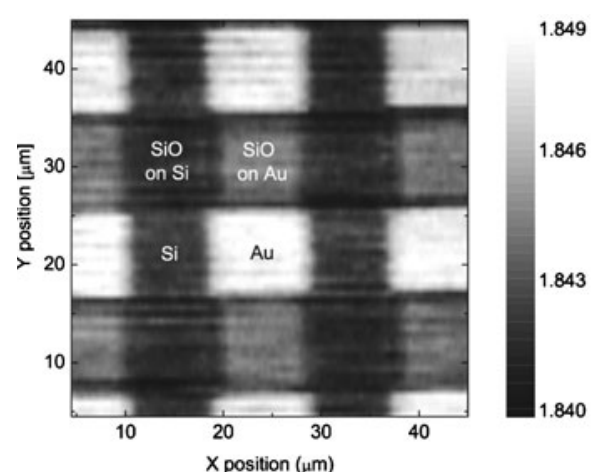

(a)

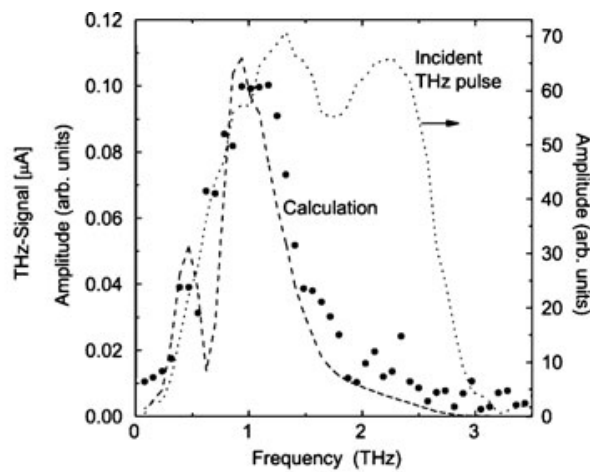

(b)

Fig. 23 a THz image of a two-dimensional semiconductor structure fabricated on semi-insulating silicon. The gold lines have vertical orientation, are $10 \mu \mathrm{m}$ wide with a thickness of $100 \mathrm{~nm}$. The $\mathrm{SiO}$ lines have horizontal orientation, are $10 \mu \mathrm{m}$ wide with a thickness of $200 \mathrm{~nm}$. b Amplitude spectrum of the incident THz pulse (dotted line), modulation signal of the THz-ANSOM (dots) via electromodulation, and model calculation of the modulation signal (dashed line) for a bulk n-doped GaAs structure described in [48]. 
Electrical failures in semiconductors Following earlier work on integrated circuits [114], Yamashita et al. demonstrated in 2008 the used of $\mathrm{THz}$ to detect electrical failures in semiconductors. To do so, they found out that $p$ - $n$ junctions can be excited by ultrafast laser pulses and, therefore, generate photocurrents which propagate along the different interconnections and produce THz pulses. They focus the beam of a Ti:Sa laser on their junction and use the same laser to detect the backward emitted $\mathrm{THz}$ radiation via a photoconductive antenna placed in the far-field. The spatial resolution is determined by the laser spot size on the junction, i.e. $25 \mu \mathrm{m}$. If the interconnection lines close to the junction, which play the role of antenna for the $\mathrm{THz}$ radiation, present some defects, the collected THz pulse will be affected. Thus, they could distinguish damaged silicon metal-oxide-semiconductors with bad connections from normal ones [115].

They improved their method by using a laser at a wavelength of $1.06 \mu \mathrm{m}$ which enables them to shine their laser beam from the backside of a largescale integrated circuit chip with a multilayered interconnection structure on top that prevents the observation from the front side [116].

Figure 24a shows illustrations of different circuits with Si p-MOSFETs; some are defective with different disconnection positions. The THz images in Fig. 24b help to distinguish a normal circuit from the defective ones: the $\mathrm{THz}$ emission signals from n-type doped areas in both areas A and B, are smaller when a disconnection occurs. These image are taken at a time delay fixed at the position indicated by the arrow in Fig. 24c.

The technique is very fast, contactless and safe for the electronics. It doesn't image the $\mathrm{THz}$ near-field itself but delivers information carried by the $\mathrm{THz}$ with an image resolution far below the wavelength. It makes a perfect example of an indirect use of local $\mathrm{THz}$ generation for inspection of larger structures.

(a)

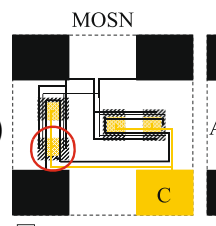

$\square$ Lightly doped area in n-type Weavily doped area in n-type W// Heavily doped area in p-type

(b)

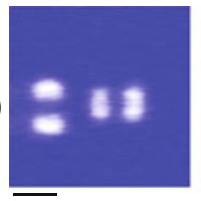

$\overline{10 \mu \mathrm{m}}$
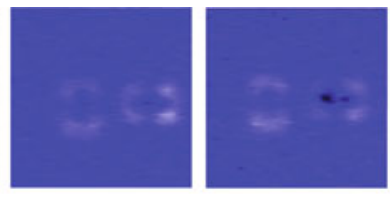

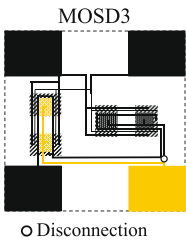

Lines and heavily doped areas in n-type electrically connected to electrode pad C

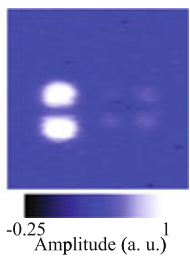

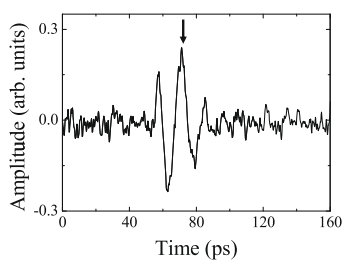

(c)

Fig. 24 a Illustrations of the MOSFETs and the defective ones (MOSD1-MOSD3) with different disconnection positions. b The corresponding $\mathrm{THz}$ emission images. $\mathbf{c}$ The waveform of the $\mathrm{THz}$ emission measured by exciting the $p$ - $n$ junctions indicated by a large circle in the MOSFET [116]. 
Metal-insulator transition $\mathrm{THz}$ near-field microscopy is also suited to study the metal-insulator transition in a vanadium dioxide $\left(\mathrm{VO}_{2}\right)$ thin film. In 2007, Mittleman's group proposed to use a vibrating ANSOM probe to investigate this effect [49]. They've deposited a $100 \mathrm{~nm}$ thin film of $\mathrm{VO}_{2}$ and applied a voltage across the sample. The ANSOM probe was placed over the sample; a $\mathrm{THz}$ beam was focus on the apex of the tip and the modulated scattered $\mathrm{THz}$ radiation could be collected in the far field. Because the local conductivity of $\mathrm{VO}_{2}$ can abruptly change when a bias is applied or when the temperature is changing, the measured $\mathrm{THz}$ will also be affected [117]. They could conclude from their measurement that micron length scale islands with different conductivity appear below the transition voltage of the film. They proved that Ohmic and non-Ohmic behavior can be probed at the micro-scale with $\mathrm{THz}$ near-field techniques.

Ferro-electric Beside probing the conductivity of a sample, these techniques can also give information on the local anisotropy in ferro-electric crystal. The group of Kadlec in Prague demonstrated this using their THz waveguide nearfield probe described above in Section 2.4 [33]. The idea is based on the strong interaction from the end of the waveguide and the sample, in their case a $\mathrm{BaTiO}_{3}$ crystal [118]. They need to perform a complex analysis derived from the "principal component analysis" to extract the information on the anisotropy changes. With the help of this analysis, they have been able to image ferromagnetic domains 5-7 $\mu \mathrm{m}$ wide, far below the size of their probe. Unfortunately the sensitivity of their probe is drastically dependent on the orientation of the probe versus the orientation of the domains due to the design of the probe. They could explain their findings with the help of simulations [34].

\subsection{Nano-spectroscopy}

As we have seen in the previous example, the near-field techniques enable to study very tiny areas at the surface of a sample or within a volume. The Planken group could perform spectroscopic analysis over a large bandwidth (0.1-7.5 THz) using their quasi near-field setup described in Section 2.7 [70]. Because the generation and the detection occurs in two different crystals facing each other in close vicinity, they could squeeze pellet of $600 \mu \mathrm{m}$ thickness in between, made of a mixture of polyethylene powder and Mannitol with different fabrication procedure [119]. They could conclude that depending on the freeze-drying technique used to produce the Mannitol, it will crystallize in different polymorph forms.

The same group imagined an other way to perform spectroscopy at the nano-volume. They use their expertise on subwavelength holes to perform their measurement: they filled a waveguide of $150 \mu \mathrm{m}$ diameter, $180 \mu \mathrm{m}$ thick, with three different samples, polyethylene powder, silicon powder and pure polycrystalline D-tartaric acid (DTA) [120]. The corresponding waveguide volume was approximately $3.2 \mathrm{nl}$. They could record deep subwavelength 

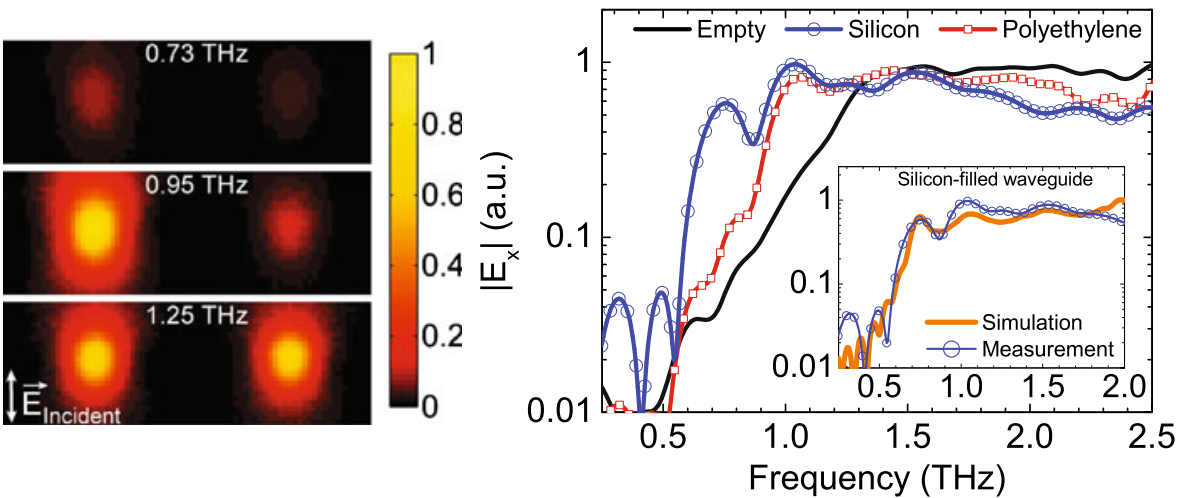

Fig. 25 Left THz electric near-field, normalized to the incoming field, as it emerges from two adjacent waveguides within an array $(P=600 \mu \mathrm{m})$. The waveguide on the left is filled with D-tartaric acid while the one on the right is empty. Note that less absorption is indicated by a darker region in the THz image. Right Spectrum, normalized to the incoming field, for siliconfilled, PE-filled and empty waveguides. The inset compares the spectrum for a silicon-filled waveguide with simulation [120].

resolution images of the light emerging from the different apertures, filled or not filled as shown in Fig. 25a. They've also collected the spectroscopic data presented in Fig. 25b, demonstrating that they could perform $\mathrm{THz}$ spectroscopy on a nano-volume of pure compounds.

These various examples confirm once more that $\mathrm{THz}$ near-field measurements can be used in a domain with immediate practical application.

\subsection{Biology}

Great hopes do exist for applying $\mathrm{THz}$ in biological applications. $\mathrm{THz}$ nearfield techniques are also moving towards biological demonstration. The extreme sensitivity to water content of the $\mathrm{THz}$ waves applied to leaves was shown by Mittleman et al. in mid 90s [121]. Schade et al., at the synchrotron facility in Berlin (BESSY) applied their conical aperture technique to image in transmission wet biological samples. An example is given in Fig. 26 with a parthenocissus leaf. They improved the resolution to $130 \mu \mathrm{m}$ at $0.36 \mathrm{THz}$ $(\lambda / 12)$. Strongly absorbing samples can be investigated with such a powerful source. The authors forecast the possibility to use this method to detect spectroscopic features of biological molecules present in organic tissue.

Bitzer et al. performed a similar experiment on a small plant leaf using their PC antenna. They gave a 2D image of the amplitude and the phase of the electric near-field for a higher frequency $(1.36 \mathrm{THz})$ reaching a $50 \mu \mathrm{m}$ resolution [76]. They could resolve sub-mm leaf veins which are typical of 200-300 $\mu \mathrm{m}$ in diameter. Their findings regarding the distribution of water in the veins raise interesting questions.

Chiu et al. in 2009 carried on in this direction of research. They used their $\mathrm{THz}$ near-field microscope based on PE fiber with a corrugated aperture 

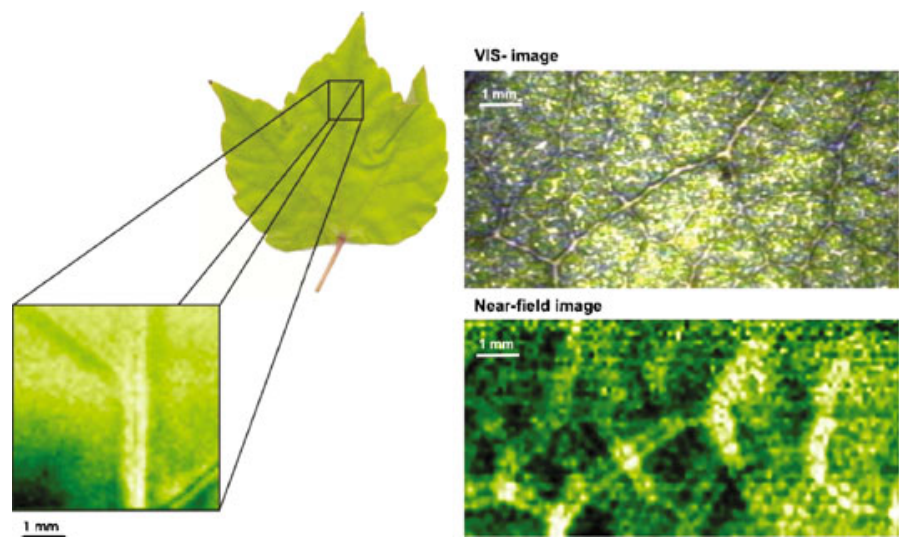

Fig. 26 Left Near-field THz image of a section of a parthenocissus leaf. Right Visible and nearfield THz image of a section of a Laurus nobilis leaf. Note that less absorption is indicated by a darker region in the THz image [122].

on biological samples [36]. It was already known that $\mathrm{THz}$ waves may help identifying between cancerous tissue and normal tissue, thanks in part to the high concentration of water around the cancerous ones [123]. But $\mathrm{THz}$ near-field techniques should bring better resolution and larger success in distinguishing between the healthy and cancerous tissues due to a better signal to noise ratio. Figure 27 presents the $\mathrm{THz}$ near-field absorption of breast tissue sections in comparison with a picture of the same breast tissue sections after staining. It shows the good agreement between the cancerous area determined by the THz near-field area and the one determined by conventional pathologic examination. The resolution obtained $(210 \mu \mathrm{m}$ for a frequency of $312 \mathrm{GHz})$ can be largely improved but it shows already the possibility of this microscope configuration.

Fig. 27 a and $\mathbf{b}$ are $\mathrm{THz}$ near-field absorption coefficient images of the breast tissue sections (samples 1 and 2). The insets show actual photographs of samples 1 and 2. $\mathbf{c}$ and $\mathbf{d}$ are photomicrographs of the breast tissue sections after H\&E staining (samples 1 and 2) [36].
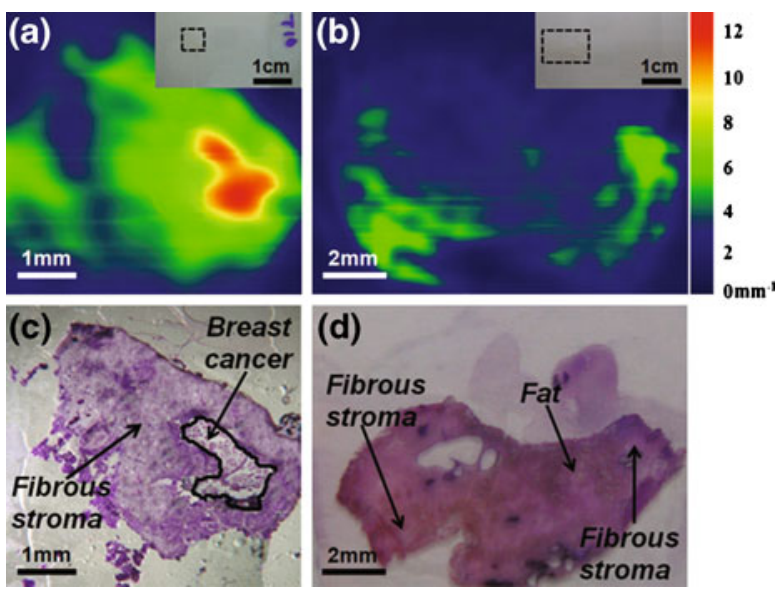


\section{Conclusion}

By the present review, we hope to have given a complete account of the latest results in the $\mathrm{THz}$ near-field domain. The resolution achieved by some of these techniques goes far beyond the simple sub-wavelength dimension, reaching the nanometer area. Other results present the advantages of simplicity with enough resolution and the broadband aspect from the Time Domain spectroscopy approach. The methods described in this paper show favorable signs of maturity which lead already to astounding applications in many different areas, from theoretical studies to inspection techniques and even potential use in biology. The techniques arrive at the critical point where they should demonstrate their unique assets versus existing technologies, especially compared to the optical domain. The availability of powerful sources, more sensitive detectors and easy system will condition its success. We believe that the THz near-field imaging is not far to breach the barriers to the commercial domain and will especially deliver significant outcomes in the study of metamaterials, inspection of devices and in nano-spectroscopy.

Acknowledgements The author would like to thanks all the researchers who have accepted to contribute by a figure to this review. A special thanks to Prof. Joseph Braat for the grammar check and Prof. Dai-Sik Kim for the help he could give. The author express also his gratitude to Prof. Planken and Prof. Martin Koch who solicited this review paper and all the various groups around the world who are performing these remarkable studies and measurements. Without them, the field and consequently the review would not have been the same.

Open Access This article is distributed under the terms of the Creative Commons Attribution Noncommercial License which permits any noncommercial use, distribution, and reproduction in any medium, provided the original author(s) and source are credited.

\section{References}

1. T. W. Ebbesen, H. J. Lezec, H. F. Ghaemi, T. Thio, and P. A. Wolff. Extraordinary optical transmission through sub-wavelenght hole arrays. Nature, 391(6668):667-669, 1998.

2. A. Lewis, M. Isaacson, A. Harootunian, and A. Muray. Development of a 500 Åspatial resolution light microscope: I. light is efficiently transmitted through $\lambda / 16$ diameter apertures. Ultramicroscopy, 13(3):227-231, 1984.

3. D. W. Pohl, W. Denk, and M. Lanz. Optical stethoscopy: Image recording with resolution $\lambda / 20$. Applied Physics Letters, 44(7):651-653, 1984.

4. Ch. Fattinger and D. Grischkowsky. Terahertz beams. Applied Physics Letters, 54(6):490492, 1989.

5. C. Kubler, R. Huber, S. Tubel, and A. Leitenstorfer. Ultrabroadband detection of multiterahertz field transients with gase electro-optic sensors: Approaching the near infrared. Applied Physics Letters, 85(16):3360-3362, 2004.

6. Alexander Sell, Alfred Leitenstorfer, and Rupert Huber. Phase-locked generation and fieldresolved detection of widely tunable terahertz pulses with amplitudes exceeding $100 \mathrm{mv} / \mathrm{cm}$. Opt. Lett., 33(23):2767-2769, Dec 2008.

7. T. Yuan, J. Z. Xu, and X. C. Zhang. Development of terahertz wave microscopes. Infrared Physics \& Technology, 45(5-6):417-425, 102004.

8. A. J. Huber, F. Keilmann, J. Wittborn, J. Aizpurua, and R. Hillenbrand. Terahertz near-field nanoscopy of mobile carriers in single semiconductor nanodevices. Nano Letters, 8(11):37663770, 2008. PMID: 18837565. 
9. A. J. L. Adam, J. M. Brok, M. A. Seo, K. J. Ahn, D. S. Kim, J. H. Kang, Q. H. Park, M. Nagel, and P. C. M. Planken. Advanced terahertz electric near-fieldmeasurements at subwavelengthdiameter metallic apertures. Opt. Express, 16(10):7407-7417, 052008.

10. Wächter M., M. Nagel, and H. Kurz. Tapered photoconductive terahertz field probe tip with subwavelength spatial resolution. Applied Physics Letters, 95:041112, 2009.

11. A. Doi, F. Blanchard, H. Hirori, and K. Tanaka. Near-field THz imaging of free induction decay from a tyrosine crystal. Opt. Express, 18(17):18419-18424, 2010.

12. Pavel Shumyatsky and Robert R. Alfano. Terahertz sources. Journal of Biomedical Optics, 16(3):033001, 2011.

13. G. B. Airy. On the Diffraction of an Object-glass with Circular Aperture. Transactions of the Cambridge Philosophical Society, 5:283, 1835.

14. Marvin Minsky. Memoir on Inventing the Confocal Scanning Microscope. Scanning, 10:128138, 1988.

15. N. Zinov'ev, A. Andrianov, A. Gallant, J. Chamberlain, and V. Trukhin. Contrast and resolution enhancement in a confocal terahertz video system. JETP Letters, 88(8):492-495, 2008.

16. E. A. Ash and G. Nicholls. Super-resolution aperture scanning microscope. Nature, 237(5357):510-512, 1972.

17. O. Mitrofanov, I. Brener, R. Harel, J.D. Wynn, L.N. Pfeiffer, K.W. West, and J. Federici. Terahertz near-field microscopy based on a collection mode detector. Applied Physics Letters, 77(22):3496-3498, 2000.

18. O. Mitrofanov, M. Lee, J. W. P. Hsu, L. N. Pfeiffer, K. W. West, J. D. Wynn, and J. F. Federici. Terahertz pulse propagation through small apertures. Applied Physics Letters, 79(7):907-909, 2001.

19. G. Gallot, S. P. Jamison, R. W. McGowan, and D. Grischkowsky. Terahertz waveguides. J. Opt. Soc. Am. B, 17(5):851-863, 2000.

20. H. A. Bethe. Theory of diffraction by small holes. Physical Review, 66(7-8), 101944.

21. C.J. Bouwkamp. On the diffraction of electromagnetic waves by small circular disk and holes. Philips Research Reports, 5:321, 1950.

22. K. Ishihara, K. Ohashi, T. Ikari, H. Minamide, H. Yokoyama, J. I. Shikata, and H. Ito. Terahertz-wave near-field imaging with subwavelength resolution using surface-waveassisted bow-tie aperture. Applied Physics Letters, 89:201120, 2006.

23. S. Mair, B. Gompf, and M. Dressel. Spatial and spectral behavior of the optical near field studied by a terahertz near-field spectrometer. Applied Physics Letters, 84(7):1219-1221, 2004.

24. Aydogan Ozcan, Ertugrul Cubukcu, Alberto Bilenca, Kenneth B. Crozier, Brett E. Bouma, Federico Capasso, and Guillermo J. Tearney. Differential near-field scanning optical microscopy. Nano Letters, 6(11):2609-2616, 102006.

25. R. Degl'Innocenti, M. Montinaro, J. Xu, V. Piazza, P. Pingue, A. Tredicucci, F. Beltram, H. E. Beere, and D. A. Ritchie. Differential near-field scanning optical microscopy with $\mathrm{THz}$ quantum cascade laser sources. Opt. Express, 17(26):23785-23792, 122009.

26. K. Ishihara, T. Ikari, H. Minamide, J.-I. Shikata, K. Ohashi, H. Yokoyama, and H. Ito. Terahertz near-field imaging using enhanced transmission through a single subwavelength aperture. Japanese Journal of Applied Physics, 44(29):L929-L931, 2005.

27. Y. Kawano and K.w Ishibashi. An on-chip near-field terahertz probe and detector. Nat Photon, 2(10):618-621, 102008.

28. S. Hunsche, M. Koch, I. Brener, and M. C. Nuss. THz near-field imaging. Optics Communications, 150(1-6):22-26, 1998.

29. T.D. Nguyen, Z.V. Vardeny, and A. Nahata. Concentration of terahertz radiation through a conically tapered aperture. Opt. Express, 18(24):25441-25448, 2010.

30. M.M. Awad and R.A. Cheville. Transmission terahertz waveguide-based imaging below the diffraction limit. Applied Physics Letters, 86(22):1-3, 2005.

31. H. Zhan, R. Mendis, and D.M. Mittleman. Superfocusing terahertz waves below $\lambda / 250$ using plasmonic parallel-plate waveguides. Opt. Express, 18(9):9643-9650, 2010.

32. G. Staats, L. Fernandez, K. Holldack, U. Schade, and D. Schondelmaier. Coupling structures for terahertz near-field microspectroscopy. Infrared Physics \& Technology, 49(1-2):122-127, 92006. 
33. N. Klein, P. Lahl, U. Poppe, F. Kadlec, and P. Kuzel. A metal-dielectric antenna for terahertz near-field imaging. Journal of Applied Physics, 98:014910, 2005.

34. M. Berta and F. Kadlec. Near-field terahertz imaging of ferroelectric domains in barium titanate. Phase Transitions: A Multinational Journal, 83(10):985-993, 2010.

35. E Kume and S Sakai. Properties of a dielectric probe for scanning near-field millimeter-wave microscopy. Journal of Applied Physics, 99(5), 2006.

36. C.-M. Chiu, H.-W. Chen, Y.-R. Huang, Y.-J. Hwang, W.-J. Lee, H.-Y. Huang, and C.-K. Sun. All-terahertz fiber-scanning near-field microscopy. Opt. Lett., 34(7):1084-1086, 2009.

37. Anastasia Rusina, Maxim Durach, Keith A. Nelson, and Mark I. Stockman. Nanoconcentration of terahertz radiation in plasmonic waveguides. Opt. Express, 16(23):18576-18589, 2008.

38. L Aigouy, FX Andreani, AC Boccara, JC Rivoal, JA Porto, R Carminati, JJ Greffet, and R Megy. Near-field optical spectroscopy using an incoherent light source. Applied Physics Letters, 76(4):397-399, 2000.

39. B. Knoll and F. Keilmann. Enhanced dielectric contrast in scattering-type scanning near-field optical microscopy. Optics Communications, 182(4-6):321-328, 82000.

40. R. Hillenbrand, T. Taubner, and F. Keilmann. Phonon-enhanced light-matter interaction at the nanometre scale. Nature, 418(6894):159-162, 072002.

41. Q. Chen, Zhiping Jiang, G. X. Xu, and X.-C. Zhang. Near-field terahertz imaging with a dynamic aperture. Opt. Lett., 25(15):1122-1124, 2000.

42. D.V. Palanker, G.M.H. Knippels, T.I. Smith, and H.A. Schwettman. Ir microscopy with a transient photo-induced near-field probe (tipless near-field microscopy). Optics Communications, 148(4-6):215-220, 1998.

43. Q. Chen and X.-C. Zhang. Semiconductor dynamic aperture for near-field terahertz wave imagin. IEEE Journal on Selected Topics in Quantum Electronics, 7(4):608-614, 2001.

44. B. Gompf, N. Gebert, H. Heer, and M. Dressel. Polarization contrast terahertz-near-field imaging of anisotropic conductors. Applied Physics Letters, 90(8), 2007.

45. N.C.J. van der Valk and P.C.M. Planken. Electro-optic detection of subwavelength terahertz spot sizes in the near field of a metal tip. Applied Physics Letters, 81(9):1558-1560, 2002.

46. H. T. Chen, R. Kersting, and G. C. Cho. Terahertz imaging with nanometer resolution. Applied Physics Letters, 83(15):3009-3011, 2003.

47. F. Zenhausern, Y. Martin, and H. K. Wickramasinghe. Scanning Interferometric Apertureless Microscopy: Optical Imaging at 10 Angstrom Resolution. Science, 269(5227):1083-1085, 1995.

48. R. Kersting, F.F. Buersgens, G. Acuna, and G.C. Cho. Terahertz near-field microscopy. Advances in Solid State Physics, 47:203-222, 2008.

49. H. Zhan, V. Astley, M. Hvasta, J.A. Deibel, D.M. Mittleman, and Y.-S. Lim. The metalinsulator transition in $\mathrm{VO}_{2}$ studied using terahertz apertureless near-field microscopy. Applied Physics Letters, 91:162110, 2007.

50. A. J. Huber, D. Kazantsev, F. Keilmann, J. Wittborn, and R. Hillenbrand. Simultaneous ir material recognition and conductivity mapping by nanoscale near-field microscopy. Advanced Materials, 19(17):2209-2212, 2007.

51. H. G. von Ribbeck, M. Brehm, D. W. van der Weide, S. Winnerl, O. Drachenko, M. Helm, and F. Keilmann. Spectroscopic THz near-field microscope. Opt. Express, 16(5):3430-3438, 032008.

52. A. Thoma and T. Dekorsy. Influence of tip-sample interaction in a time-domain terahertz scattering near field scanning microscope. Applied Physics Letters, 92(25), 2008.

53. T. Yuan, H. Park, J. Xu, H. Han, and X.-C. Zhang. THz wave near-field emission microscope. Ultrafast Phenomena XIV, 79:759-761, 2005.

54. K.L. Wang and D.M. Mittleman. Metal wires for terahertz wave guiding. Nature, 432(7015):376-379, 2004.

55. R. Adam, L. Chusseau, T. Grosjean, A. Penarier, J.-P. Guillet, and D. Charraut. Near-field wire-based passive probe antenna for the selective detection of the longitudinal electric field at terahertz frequencies. Journal of Applied Physics, 106(7), 2009.

56. M. Awad, M. Nagel, and H. Kurz. Tapered sommerfeld wire terahertz near-field imaging. Applied Physics Letters, 94(5), 2009.

57. Y. Bin Ji, E.S. Lee, J.S. Jang, and T.I. Jeon. Enhancement of the detection of THz sommerfeld wave using a conical wire waveguide. Opt. Express, 16(1):271-278, 2008. 
58. H. Liang, S. Ruan, and M. Zhang. Terahertz surface wave propagation and focusing on conical metal wires. Opt. Express, 16(22):18241-18248, 2008.

59. Xiao-Yong He. Investigation of terahertz sommerfeld wave propagation along conical metal wire. J. Opt. Soc. Am. B, 26(9):A23-A28, 2009.

60. R. Lecaque, S. Grésillon, N. Barbey, R. Peretti, J.-C. Rivoal, and C.w Boccara. THz near-field optical imaging by a local source. Optics Communications, 262(1):125-128, 2006.

61. M. Yi, K. Lee, J. Lim, Y. Hong, Y.-D. Jho, and J. Ahn. Terahertz waves emitted from an optical fiber. Opt. Express, 18(13):13693-13699, 2010.

62. R. Lecaque, S.l Grésillon, and C. Boccara. THz emission microscopy with sub-wavelength broadband source. Opt. Express, 16(7):4731-4738, 2008.

63. Andreas Gürtler, Carsten Winnewisser, Hanspeter Helm, and Peter Uhd Jepsen. Terahertz pulse propagation in the near field and the far field. J. Opt. Soc. Am. A, 17(1):74-83, 2000.

64. K Yang, LPB Katehi, and JF Whitaker. Electro-optic field mapping system utilizing external gallium arsenide probes. Applied Physics Letters, 77(4):486-488, 2000.

65. S. Seitz, M. Bieler, G. Hein, K. Pierz, U. Siegner, F. J. Schmueckle, and W. Heinrich. Characterization of an external electro-optic sampling probe: Influence of probe height on distortion of measured voltage pulses. Journal of Applied Physics, 100(11), 2006.

66. Nick C. J. van der Valk, Tom Wenckebach, and Paul C. M. Planken. Full mathematical description of electro-optic detection in optically isotropic crystals. J. Opt. Soc. Am. B, 21(3):622-631, 2004.

67. M. A. Seo, A. J. L. Adam, J. H. Kang, J. W. Lee, S. C. Jeoung, Q. H. Park, P. C. M. Planken, and D. S. Kim. Fourier-transform terahertz near-field imaging of one-dimensional slit arrays: mapping of electric-field-, magnetic-field-, and poynting vectors. Opt. Express, 15(19):11781$11789,092007$.

68. A.J.L. Adam, J.M. Brok, P.C.M. Planken, M.A. Seo, and D.S. Kim. THz near-field measurements of metal structures. Comptes Rendus Physique, 9(2):161-168, 2008.

69. A. Bitzer and M. Walther. Terahertz near-field imaging of metallic subwavelength holes and hole arrays. Applied Physics Letters, 92:231101, 2008.

70. R. Chakkittakandy, J.A. Corver, and P.C.M. Planken. Quasi-near field terahertz generation and detection. Opt. Express, 16(17):12794-12805, 082008.

71. O Mitrofanov. Tetrahertz near-field electro-optic probe based on a microresonator. Applied Physics Letters, 88:091118, 2006.

72. Chao-Chun Chen. Transmission of microwave through perforated flat plates of finite thickness. IEEE Transactions on Microwave Theory and Techniques, 21(1):1-6, Jan 1973.

73. J. Gómez Rivas, C. Schotsch, P. Haring Bolivar, and H. Kurz. Enhanced transmission of THz radiation through subwavelength holes. Physical Review B, 68(20), 112003.

74. J. R. Knab, A. J. L. Adam, M. Nagel, E. Shaner, M. A. Seo, D. S. Kim, and P. C. M. Planken. Terahertz near-field vectorial imaging of subwavelength apertures and aperture arrays. Opt. Express, 17(17):15072-15086, 082009.

75. L. Guestin, A. J. L. Adam, J. R. Knab, M. Nagel, and P. C. M. Planken. Influence of the dielectric substrateon the terahertz electric near-fieldof a hole in a metal. Opt. Express, 17(20):17412-17425, 092009.

76. A. Bitzer, A. Ortner, and M. Walther. Terahertz near-field microscopy with subwavelength spatial resolution based on photoconductive antennas. Appl. Opt., 49(19):E1-E6, 07 2010.

77. F. J. García-Vidal, Esteban Moreno, J. A. Porto, and L. Martín-Moreno. Transmission of light through a single rectangular hole. Physical Review Letters, 95(10), 082005.

78. M. A. Seo, A. J. L. Adam, J. H. Kang, J. W. Lee, K. J. Ahn, Q. H. Park, P. C. M. Planken, and D. S. Kim. Near field imaging of terahertz focusing onto rectangular apertures. Opt. Express, 16(25):20484-20489, 122008.

79. Seo M. A., Park H. R., Koo S. M., Park D. J., Kang J. H., Suwal O. K., Choi S. S., Planken P. C. M., Park G. S., Park N. K., Park Q. H., and Kim D. S. Terahertz field enhancement by a metallic nano slit operating beyond the skin-depth limit. Nat Photon, 3(3):152-156, 03 2009.

80. H. R. Park, Y. M. Park, H. S. Kim, J. S. Kyoung, M. A. Seo, D. J. Park, Y. H. Ahn, K. J. Ahn, and D. S. Kim. Terahertz nanoresonators: Giant field enhancement and ultrabroadband performance. Applied Physics Letters, 96:12110, 2010. 
81. A. Bitzer, M. Walther, A. Kern, S. Gorenflo, and H. Helm. Examination of the spatial and temporal field distributions of single-cycle terahertz pulses at a beam focus. Applied Physics Letters, 90:071112, 2007.

82. A. Bitzer, H. Helm, and M. and Walther. Beam-profiling and wavefront-sensing of $\mathrm{THz}$ pulses at the focus of a substrate-lens. IEEE Journal on Selected Topics in Quantum Electronics, 14(2):476-481, 2008.

83. S. Winnerl, B. Zimmermann, F. Peter, H. Schneider, and M. Helm. Terahertz bessel-gauss beams of radial and azimuthal polarization from microstructured photoconductive antennas. Opt. Express, 17(3):1571-1576, 2009.

84. A. Bitzer, H. Merbold, A. Thoman, T. Feurer, H. Helm, and M. Walther. Terahertz nearfield imaging of electric and magnetic resonances of a planar metamaterial. Opt. Express, 17(5):3826-3834, 032009.

85. J. B. Pendry, D. Schurig, and D. R. Smith. Controlling Electromagnetic Fields. Science, 312(5781):1780-1782, 2006.

86. Nikolay I. Zheludev. The Road Ahead for Metamaterials. Science, 328(5978):582-583, 2010.

87. Andreas Bitzer, Alex Ortner, Hannes Merbold, Thomas Feurer, and Markus Walther. Terahertz near-field microscopy of complementary planar metamaterials: Babinet's principle. Opt. Express, 19(3):2537-2545, 2011.

88. J. A. Riordan, F. G. Sun, Z. G. Lu, and X. C. Zhang. Free-space transient magneto-optic sampling. Applied Physics Letters, 71(11):1452-1454, 1997.

89. M. Burresi, D. van Oosten, T. Kampfrath, H. Schoenmaker, R. Heideman, A. Leinse, and L. Kuipers. Probing the Magnetic Field of Light at Optical Frequencies. Science, 326(5952):550-553, 2009.

90. G. Acuna, S. F. Heucke, F. Kuchler, H. T. Chen, A. J. Taylor, and R. Kersting. Surface plasmons in terahertz metamaterials. Opt. Express, 16(23):18745-18751, 2008.

91. J. Zenneck. Fortplfanzung ebener elektromagnetischer wellen längs einer ebenen leiterfläche. Annals of Physics, 23:846, 1907.

92. A. Sommerfeld. Ueber die fortpflanzung elektrodynamischer wellen längs eines drahtes. Ann. Phys. Chem., 67:233, 1899.

93. T.-I Jeon and D.a Grischkowsky. THz zenneck surface wave (THz surface plasmon) propagation on a metal sheet. Applied Physics Letters, 88(6), 2006.

94. J. Saxler, J. Gómez Rivas, C. Janke, H. P. M. Pellemans, P. Haring Bolívar, and H. Kurz. Time-domain measurements of surface plasmon polaritons in the terahertz frequency range. Physical Review B, 69:15542, 2004.

95. John O'Hara, Richard Averitt, and Antoinette Taylor. Prism coupling to terahertz surface plasmon polaritons. Opt. Express, 13(16):6117-6126, 2005.

96. Wenqi Zhu and Ajay Nahata. Electric field vector characterization of terahertz surface plasmons. Opt. Express, 15(9):5616-5624, 2007.

97. Wenqi Zhu, Amit Agrawal, and Ajay Nahata. Direct measurement of the gouy phase shift for surface plasmon-polaritons. Opt. Express, 15(16):9995-10001, 2007.

98. J. B. Pendry, L. Martín-Moreno, and F. J. Garcia-Vidal. Mimicking Surface Plasmons with Structured Surfaces. Science, 305(5685):847-848, 2004.

99. J. Gómez Rivas, M. Kuttge, P. Haring Bolivar, H. Kurz, and J. A. Sánchez-Gil. Propagation of surface plasmon polaritons on semiconductor gratings. Physical Review Letters, 93:256804, 2004.

100. M. Wächter, M. Nagel, and H. Kurz. Frequency-dependent characterization of THz sommerfeld wave propagation on single-wires. Opt. Express, 13(26):10815-10822, 2005.

101. V. Astley, R. Mendis, and D.M. Mittleman. Characterization of terahertz field confinement at the end of a tapered metal wire waveguide. Applied Physics Letters, 95:031104, 2009.

102. Stefan A. Maier, Steve R. Andrews, L. Martín-Moreno, and F. J. García-Vidal. Terahertz surface plasmon-polariton propagation and focusing on periodically corrugated metal wires. Phys. Rev. Lett., 97(17):176805, 2006.

103. O. Mitrofanov, T. Tan, P. R. Mark, B. Bowden, and J. A. Harrington. Waveguide mode imaging and dispersion analysis with terahertz near-field microscopy. Applied Physics Letters, 94, 1711042009. 
104. K. Nielsen, H.K. Rasmussen, A.J.L. Adam, P.C.M. Planken, O. Bang, and P.U. Jepsen. Bendable, low-loss topas fibers for the terahertz frequency range. Opt. Express, 17(10):85928601, 2009.

105. M. L. M. Balistreri, H. Gersen, J. P. Korterik, L. Kuipers, and N. F. van Hulst. Tracking femtosecond laser pulses in space and time. Science, 294(5544):1080-1082, 2001.

106. D. Martin-Cano, M. L. Nesterov, A. I. Fernandez-Dominguez, F. J. Garcia-Vidal, L. MartinMoreno, and Esteban Moreno. Domino plasmons for subwavelengthterahertz circuitry. Opt. Express, 18(2):754-764, 2010.

107. K.L. Wang, D.M. Mittleman, N.C.J. van der Valk, and P.C.M. Planken. Antenna effects in terahertz apertureless near-field optical microscopy. Applied Physics Letters, 85(14):27152717, 2004.

108. A.J.L. Adam, N.C. van der Valk, and P.C.M. Planken. Measurement and calculation of the near field of a terahertz apertureless scanning optical microscope. J. Opt. Soc. Am. B, 24(5):1080-1090, 052007.

109. H.-T. Chen, S. Kraatz, G. C. Cho, and R. Kersting. Identification of a resonant imaging process in apertureless near-field microscopy. Phys. Rev. Lett., 93(26):267401, 2004.

110. A. Cvitkovic, N. Ocelic, and R. Hillenbrand. Analytical model for quantitative prediction of material contrasts inscattering-type near-field optical microscopy. Opt. Express, 15(14):85508565, 2007.

111. V. Astley, H. Zhan, R. Mendis, and Daniel M. Mittleman. A study of background signals in terahertz apertureless near-field microscopy and their use for scattering-probe imaging. Journal of Applied Physics, 95:031104, 2009.

112. F. Buersgens, R. Kersting, and H. T. Chen. Terahertz microscopy of charge carriers in semiconductors. Applied Physics Letters, 88:112115, 2006.

113. F. Buersgens, G. Acuna, C. H. Lang, S. I. Potrebic, S. Manus, and R. Kersting. Shear force control for a terahertz near field microscope. Review of Scientific Instruments, 78:113701, 2007.

114. T. Kiwa, M. Tonouchi, M. Yamashita, and K. Kawase. Laser terahertz-emission microscope for inspecting electrical faults in integrated circuits. Opt. Lett., 28(21):2058-2060, 112003.

115. M. Yamashita, C. Otani, K. Kawase, K. Nikawa, and M.w Tonouchi. Noncontact inspection technique for electrical failures in semiconductor devices using a laser terahertz emission microscope. Applied Physics Letters, 93:041117, 2008.

116. M. Yamashita, C. Otani, K. Kawase, T. Matsumoto, K. Nikawa, S. Kim, H. Murakami, and M. Tonouchi. Backside observation of large-scale integrated circuits with multilayered interconnections using laser terahertz emission microscope. Applied Physics Letters, 94:191104, 2009.

117. P.U. Jepsen, Bernd M. Fischer, A. Thoman, H. Helm, J. Y. Suh, R. Lopez, and R. F. Haglund.

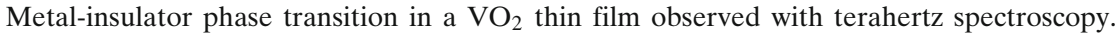
Physical Review B, 74:205103, 2006.

118. M. Berta, P. Kužel, and F. Kadlec. Study of responsiveness of near-field terahertz imaging probes. Journal of Physics D: Applied Physics, 42(15):155501, 2009.

119. R. Chakkittakandy, J.A. Corver, and P.C.M. Planken. Terahertz spectroscopy to identify the polymorphs in freeze-dried mannitol. Journal of Pharmaceutical Sciences, 99(2):932-940, 2010 .

120. J.R. Knab, A.J.L. Adam, R. Chakkittakandy, and P.C.M. Planken. Terahertz near-field microspectroscopy. Applied Physics Letters, 97(3):031115, 2010.

121. D.M. Mittleman, R.H. Jacobsen, and M.C. Nuss. T-ray imaging. IEEE Journal on Selected Topics in Quantum Electronics, 2(3):679-692, 1996.

122. U. Schade, K. Holldack, P. Kuske, G. Wüstefeld, and H. W. Hübers. THz near-field imaging employing synchrotron radiation. Applied Physics Letters, 84(8):1422-1424, 2004.

123. P.C. Ashworth, E. Pickwell-MacPherson, E. Provenzano, S.E. Pinder, A.D. Purushotham, M. Pepper, and V.P. Wallace. Terahertz pulsed spectroscopy of freshly excised human breast cancer. Opt. Express, 17(15):12444-12454, 2009. 